\title{
Ginsenoside Rh2 stimulates the production of mitochondrial reactive oxygen species and induces apoptosis of cervical cancer cells by inhibiting mitochondrial electron transfer chain complex
}

\author{
YING LIU $^{1 *}$, SHITING YU ${ }^{1 *}$, XIN XING ${ }^{1}$, JUHUI QIAO ${ }^{1}$, YIQIU YIN $^{2}$, \\ JIAWEN WANG ${ }^{1}$, MEICHEN LIU ${ }^{1}$ and WEI ZHANG ${ }^{3}$ \\ ${ }^{1}$ Jilin Ginseng Academy, Changchun University of Chinese Medicine, Changchun, Jilin 130117; \\ ${ }^{2}$ GeneScience Pharmaceuticals Co., Ltd., Changchun, Jilin 130012; ${ }^{3}$ Scientific Research Department, \\ Changchun University of Chinese Medicine, Changchun, Jilin 130117, P.R. China
}

Received June 25, 2021; Accepted October 7, 2021

DOI: $10.3892 / \mathrm{mmr} .2021 .12513$

\begin{abstract}
Ginsenoside Rh2 (G-Rh2) is a monomeric compound that extracted from ginseng and possesses anti-cancer activities both in vitro and in vivo. Previously, we reported that G-Rh2 induces apoptosis in HeLa cervical cancer cells and that the process was related to reactive oxygen species (ROS) accumulation and mitochondrial dysfunction. However, the upstream mechanisms of G-Rh2, along with its cellular targets, remain to be elucidated. In the present study, the Cell Counting Kit- 8 assay, flow cytometry and Hoechst staining revealed that G-Rh2 significantly inhibited cell viability and induced apoptosis of cervical cancer cells. However, G-Rh2 was demonstrated to be
\end{abstract}

Correspondence to: Dr Meichen Liu, Jilin Ginseng Academy, Changchun University of Chinese Medicine, 1035 Boshuo Road, Nanguan, Changchun, Jilin 130117, P.R. China

E-mail: liumc0367@163.com

Mr. Wei Zhang, Scientific Research Department, Changchun University of Chinese Medicine, 1035 Boshuo Road, Nanguan, Changchun, Jilin 130117, P.R. China

E-mail: weizcaas@126.com

*Contributed equally

Abbreviations: G-Rh2, ginsenoside Rh2; MMP, mitochondrial membrane potential; ETC, electron transport chain; mtROS, mitochondrial reactive oxygen species; CCK-8, Cell Counting Kit-8; OCR, oxygen consumption rate; FCCP, carbonyl cyanide-4-(trifluoromethoxy) phenylhydrazone; ECAR, extracellular acidification rate; OXPHOS, oxidative phosphorylation; NDUFS1, NADH: ubiquinone oxidoreductase core subunit S1; UQCRC1, biquinol-cytochrome c reductase core protein 1; ATP5F1B, ATP synthase F1 subunit beta

Key words: ginsenoside Rh2, mitochondria, oxidative phosphorylation, reactive oxygen species, mitochondrial electron transfer chain complexes non-toxic to End1/e6e7 cells. JC-1, rhodamine 123 staining, oxidative phosphorylation and glycolysis capacity assays demonstrated that G-Rh2 exposure caused an immediate decrease in mitochondrial transmembrane potential due to its inhibition of mitochondrial oxidative phosphorylation, as well as glycolysis, both of which reduced cellular ATP production. Western blotting and electron transport chain (ETC) activity assays revealed that G-Rh2 significantly inhibited the activity of ETC complexes I, III and V. Overexpression of ETC complex III partially significantly restored mitochondrial ROS and inhibited the apoptosis of cervical cancer cells induced by G-Rh2. The predicted results of binding energy in molecular docking, confirmed that G-Rh2 was highly likely to induce mitochondrial ROS production and promote cell apoptosis by targeting the ETC complex, especially for ETC complex III. Taken together, the present results revealed the potential anti-cervical cancer activity of G-Rh2 and provide direct evidence for the contribution of impaired ETC complex activity to cervical cancer cell death.

\section{Introduction}

Cervical cancer is one of the most common malignancies in females worldwide (1). Surgery and concurrent chemoradiotherapy are the two primary treatment options for patients with cervical cancer (2). Traditional chemotherapy drugs cause serious adverse reactions and thus, the development of more effective therapeutics will provide a marked benefit for patients with cervical cancer (3).

Ginsenoside Rh2 (G-Rh2) is a major bioactive component of ginseng (4) that has been indicated to significantly inhibit the proliferation of cancer cells in cell culture and in tumor models of various human cancers (5-8). Previous studies have indicated that apoptosis of cancer cells induced by G-Rh2 is caused by an excessive accumulation of mitochondrial reactive oxygen species (mtROS), accompanied by the occurrence of numerous downstream apoptotic events, such as mitochondrial depolarization, cytochrome c release and activation of caspase enzymes (9-11). However, the upstream mechanisms through 
which G-Rh2 induces mtROS-mediated apoptosis have remained elusive.

Recently, numerous studies have demonstrated that mitochondrial dysfunction resulting in mtROS overproduction is a potential cause of cancer cell apoptosis (12-14). Mitochondria perform oxidative phosphorylation (OXPHOS) to synthesize ATP through the mitochondrial electron transport chain (ETC), which comprises complexes I-V. The leakage of electrons from the gradient formed by the ETC complexes in the electron transfer process is the main source of mtROS (15-17). As an unavoidable byproduct of metabolism, the mtROS concentration is normally maintained at a low level by the corresponding scavenger system and thereby a steady state is maintained, which is important because mtROS are a significant signal transducer for various molecular pathways (18). However, if an imbalance or decompensation of mtROS production/scavenging occurs, cells become damaged $(19,20)$ with reduced mitochondrial function and they subsequently undergo apoptosis (21-23). In addition, a recent study by our group reported that G-Rh2 induces apoptosis in HeLa cervical cancer cells and that the process was related to ROS accumulation and mitochondrial dysfunction (24). Based on these previous findings, it was hypothesized that G-Rh2 targets ETC complexes, which mediates the generation of mtROS and induces apoptosis of cervical cancer cells. The aim of the present study was to further investigate the novel upstream direct target of mtROS production and apoptosis induction by G-Rh2. The present study provided novel insight into how G-Rh2 induces apoptosis in cervical cancer cells.

\section{Materials and methods}

Cell culture. The human cervical cancer cell lines HeLa [human papillomavirus (HPV)-positive] and C33A (HPV-negative) were obtained from the Type Culture Collection of the Chinese Academy of Sciences and cultured in DMEM supplemented with $10 \%$ fetal bovine serum, $100 \mathrm{U} / \mathrm{ml}$ penicillin and $100 \mu \mathrm{g} / \mathrm{ml}$ streptomycin (all from Hyclone; Cytiva). The nontumorigenic (control) End1/e6e7 cell line was purchased from the American Type Culture Collection and cultured in KSFM medium (Gibco; Thermo Fisher Scientific, Inc.) with bovine pituitary extract $(0.05 \mathrm{mg} / \mathrm{ml})$, human recombinant epidermal growth factor $(0.1 \mathrm{ng} / \mathrm{ml})$, calcium chloride $(44.1 \mathrm{mg} / \mathrm{l})$ (all from Shanghai Absin Biotechnology Co., Ltd) and $1 \%$ antibiotics (100 U/ml penicillin and $100 \mu \mathrm{g} / \mathrm{ml}$ streptomycin; Hyclone; Cytiva). All cell lines were cultured in a humidified normoxic chamber (Thermo Fisher Scientific, Inc.) with $5 \% \mathrm{CO}_{2}$ at $37^{\circ} \mathrm{C}$.

Assessment of cell viability. HeLa, C33A and End1/e6e7 cells $\left(2 \times 10^{4}\right.$ cells/well) were inoculated onto 96 -well plates and incubated for $24 \mathrm{~h}$. To determine the $50 \%$ growth inhibition concentration $\left(\mathrm{IC}_{50}\right)(25,26)$, cells were treated with 35, 45, 55 and $65 \mu \mathrm{M}$ G-Rh2 (Yuanye Biotechnology Co., Ltd) for $24 \mathrm{~h}$. Cell viability was measured using the Cell Counting Kit-8 (CCK-8) assay (Wuhan Boster Biological Technology, Ltd.) according to the manufacturer's protocol. Evaluation was performed using an Infinite M200 PRO plate reader (Tecan Group, Ltd.) at $450 \mathrm{~nm}$.
Flow cytometric analysis of cell death mode. The mode of cell death was assessed using an FITC Annexin V Apoptosis Detection Kit I (BD Biosciences). Cells were inoculated onto 6-well plates and incubated for $24 \mathrm{~h}$. HeLa cells were treated with 35 and $45 \mu \mathrm{M} \mathrm{G}-\mathrm{Rh} 2$ for $24 \mathrm{~h}$ and C33A cells were treated with 45 and $55 \mu \mathrm{M} \mathrm{G}-\mathrm{Rh} 2$ for $24 \mathrm{~h}$. After treatment, the cells were collected by centrifugation, washed twice with ice-cold PBS, suspended in 1X Binding Buffer and then incubated with $5 \mu 1$ each of propidium iodide (PI) and Annexin V-FITC solution. Probes were incubated at $37^{\circ} \mathrm{C}$ in the dark for $15 \mathrm{~min}$. Samples were examined with a flow cytometer (Amnis Corporation) and quantified using IDEAS software v6.1 (Amnis Corporation).

Apoptosis morphology detection with Hoechst 33342 staining. The mode of cell death was also demonstrated by Hoechst 33342 staining. After treating cells as above, the culture medium was removed and cells were washed twice with PBS and then submerged in $1 \mathrm{ml}$ Hoechst 33342 staining solution (Beyotime Institute of Biotechnology). The cells were washed twice again after labelling for $30 \mathrm{~min}$ at $37^{\circ} \mathrm{C}$ in the dark and then observed with the EVOS FL Auto Imaging System (Thermo Fisher Scientific, Inc.).

Determination of mitochondrial membrane potential (MMP). JC-1 (Beijing Solarbio Science and Technology, Co., Ltd.) and rhodamine 123 (Beyotime Institute of Biotechnology) were used to evaluate the MMP. HeLa cells were treated with G-Rh2 at 35 and $45 \mu \mathrm{M}$ for $24 \mathrm{~h}$ and C33A cells were treated with G-Rh2 at 45 and $55 \mu \mathrm{M}$ for $24 \mathrm{~h}$. After removing the medium and washing the cells twice with cold PBS, $1 \mathrm{ml}$ of cell culture medium supplemented with $1 \mathrm{ml} \mathrm{JC}-1$ dye working solution was added, followed by incubation at $37^{\circ} \mathrm{C}$ in the dark for $20 \mathrm{~min}$. Cells were then washed twice with JC-1 staining buffer prior to imaging with the EVOS FL Auto Imaging System (Thermo Fisher Scientific, Inc.). Rhodamine 123 was also used to estimate changes in MMP. In brief, cells were stained with rhodamine 123 in the dark at $37^{\circ} \mathrm{C}$ for $30 \mathrm{~min}$. Cells were washed twice with PBS prior to loading and evaluation by flow cytometry (Amnis Corporation).

Analysis of intracellular ATP levels. An ATP analysis kit (Beyotime Institute of Biotechnology) was used to detect intracellular ATP levels. In brief, cells inoculated onto 6-well cell culture plates were incubated for $24 \mathrm{~h}$ and subsequently, HeLa cells were treated with G-Rh2 at 35 and $45 \mu \mathrm{M}$ for $24 \mathrm{~h}$ and C33A cells were treated with G-Rh2 at 45 and $55 \mu \mathrm{M}$ for $24 \mathrm{~h}$. The culture medium was then removed and replaced with $200 \mu 1$ ice-cold ATP lysis buffer and the lysate was then centrifuged at $12,000 \mathrm{xg}$ for $10 \mathrm{~min}$ at $4^{\circ} \mathrm{C}$. The supernatant was then transferred to a new tube. The reaction was initiated at $37^{\circ} \mathrm{C}$ for 3 min to consume background ATP with $100 \mu \mathrm{l}$ ATP detection buffer prior to adding $10 \mu 1$ supernatant for detection. Optical density values were recorded using an Infinite M200 PRO plate reader (Tecan Group, Ltd.) and the ATP content was converted according to the standard curve.

Measurement of oxygen consumption rate (OCR). The OCR was tested using the Seahorse Bioscience XFp Extracellular Flux Analyzer (Agilent Technologies, Inc.) according to the 
manufacturer's protocol. The Seahorse XFp Mito Stress Test Kit (Agilent Technologies, Inc.) was applied to measure the OCR. After treatment with G-Rh2 (35 and $45 \mu \mathrm{M})$ for $24 \mathrm{~h}$, HeLa cells $\left(1 \times 10^{4} /\right.$ well $)$ were inoculated onto XFp cell culture plates for $24 \mathrm{~h}$. The cells were then washed with analysis medium (supplemented with $10 \mathrm{mM}$ glucose, $2 \mathrm{mM}$ glutamine and $1 \mathrm{mM}$ sodium pyruvate). Subsequently, the plates were placed in a non- $\mathrm{CO}_{2}$ incubator at $37^{\circ} \mathrm{C}$ for at least $1 \mathrm{~h}$ for degassing. After baseline measurement, $1 \mu \mathrm{M}$ oligomycin (ATP synthase inhibitor), $1 \mu \mathrm{M}$ carbonyl cyanide-4-(trifluoromethoxy) phenylhydrazone (FCCP; uncoupler) and $0.5 \mu \mathrm{M}$ rotenone (rot)/antimycin A (AA) mixture (inhibitors of ETC complexes I/III) were used to perform real-time OCR quantifications. Data were evaluated using Wave software version 2.6.3 (Agilent Technologies, Inc.) and OCR was expressed in $\mathrm{pmol} / \mathrm{min}$.

Measurement of extracellular acidification rate (ECAR). The ECAR was detected using the Seahorse XF Glycolysis Stress Test Kit (Agilent Technologies, Inc.) on the Seahorse XFp Extracellular Flux Analyzer (Agilent Technologies, Inc.) according to the manufacturer's protocol. In brief, HeLa cells were treated with 35 or $45 \mu \mathrm{M}$ G-Rh2 for $24 \mathrm{~h}$ and then inoculated onto Seahorse XFp cell culture microtiter plates for $24 \mathrm{~h}\left(1 \times 10^{4}\right.$ cells/well). The cells were then washed with assay medium (supplemented with $2 \mathrm{mM}$ glutamine) prior to degassing treatment at $37^{\circ} \mathrm{C}$ in a non- $\mathrm{CO}_{2}$ incubator for $1 \mathrm{~h}$. After the ECAR baseline was calibrated, $10 \mathrm{mM}$ glucose, $1 \mu \mathrm{M}$ oligomycin (OXPHOS inhibitor) and $50 \mathrm{mM}$ 2-deoxy-d-glucose (glycolysis inhibitor) were injected into the detection wells at the specified time points. Data were evaluated using Wave software version 2.6.3 (Agilent Technologies, Inc.) and ECAR was expressed in $\mathrm{mpH} / \mathrm{min}$.

Cell fractionation. The Cell Mitochondrial Isolation Kit (Beyotime Institute of Biotechnology) was used to extract mitochondrial protein. HeLa cells were collected and rinsed with cold PBS after treatment with 35 and $45 \mu \mathrm{M}$ G-Rh2 for $24 \mathrm{~h}$, and then treated with ice-cold mitochondrial separation reagent containing $1 \mathrm{mM}$ PMSF for $10 \mathrm{~min}$. Next, the cells were homogenized and the supernatant was centrifuged at $11,000 \mathrm{x} \mathrm{g}$ for $10 \mathrm{~min}$ at $4^{\circ} \mathrm{C}$. The mitochondrial protein precipitate was lysed with mitochondrial lysate for subsequent western blotting analysis. Finally, total protein was obtained by lysing HeLa cells with RIPA buffer (Beyotime Institute of Biotechnology). The protein concentration of the samples was detected using a BCA Protein Assay Kit.

Western blot analysis. Western blot was applied to analyze protein expression. In brief, denatured protein samples $(30 \mu \mathrm{g})$ were separated by $12 \%$ SDS-PAGE, transferred to PVDF membranes (EMD Millipore), blocked in 5\% skimmed milk (Beijing Solarbio Science \& Technology, Co., Ltd.) for $2 \mathrm{~h}$ at room temperature, washed with PBS containing Tween-20 (PBST) and incubated with primary antibodies [total OXPHOS human WB antibody cocktail (cat no. ab110411); anti-myc tag (cat no. ab206486); anti-Flag (cat no. ab205606); and anti-6xHis tag (cat no. ab18184); all 1:1,000 dilution; Abcam] at $4^{\circ} \mathrm{C}$ overnight on an orbital shaker. PVDF membranes were then washed with PBST and incubated with horseradish peroxidase-conjugated anti-mouse IgG (cat no. sc-2005; 1:1,000 dilution; Santa Cruz Biotechnology, Inc.) at room temperature for $1 \mathrm{~h}$ prior to washing with PBST and adding a working solution of Enhanced Chemiluminescence Reagent Kit (Beyotime Institute of Biotechnology). Membranes were imaged using the iBright FL1000 Imaging System (Invitrogen; Thermo Fisher Scientific, Inc.). Image J software 1.53a (National Institutes of Health) was used to quantify the gray value of western blot bands and the protein expression level was normalized as the gray value of the target protein/the loading control protein.

ETC complex activity assay. ETC complex activity detection kits (cat no. BC0515, BC3235, BC3245, BC0945 and BC1445; Beijing Solarbio Science \& Technology, Co., Ltd.) were used to measure the enzymatic activity of ETC complexes I-V with an Infinite M200 PRO Plate Reader (Tecan Group, Ltd.) according to the manufacturer's protocol. The oxidation rate of $\mathrm{NADH}$ was recorded at $340 \mathrm{~nm}$ to assess the activity of ETC complex I. The rate of 2,6-dichloroindolephenol reduction was used to measure the activity of ETC complex II at $605 \mathrm{~nm}$. The activity of ETC complex III was detected by measuring the increase rate of the light absorption of cytochrome c-reduced at $550 \mathrm{~nm}$. The activity of ETC complex IV was monitored by measuring the rate of decrease in the absorbance of reduced cytochrome $\mathrm{c}$ at $550 \mathrm{~nm}$. The activity of ETC complex V was quantified by calculating the rate of addition of inorganic phosphate (Pi) at $660 \mathrm{~nm}$.

Molecular docking. Molecular docking analyses were performed for G-Rh2 and the ETC complexes. Data on the structures of ETC complexes were obtained from the RCSB Protein Data Bank (PDB; http://www.rcsb.org/pdb), including ETC complex I (PDB ID: 4G73) (27), ETC complex III (PDB ID: 4PD4) (28) and ETC complex V (1BMF) $(29,30)$. Molecular docking was performed with AutoDock tools (version 4.2.6) and visualized with the Discovery Studio 4.0 Visualizer (BIOVIA).

Measurement of mtROS. The mitoSOX probe (Beyotime Institute of Biotechnology) was used to determine mtROS production. First, cells were seeded onto 6-well cell culture plates. After $24 \mathrm{~h}$ of incubation, G-Rh2 specificity was determined by pretreating HeLa cells with mitoQ $(5 \mu \mathrm{M})$ for $1 \mathrm{~h}$ prior to treatment with G-Rh2 (35 and $45 \mu \mathrm{M})$ for $24 \mathrm{~h}$. The cells were collected by centrifugation and washed with cold PBS prior to adding $5 \mu \mathrm{M}$ mitoSOX probe (cat. no. M36008; Invitrogen; Thermo Fisher Scientific, Inc.) and incubating the mixture at $37^{\circ} \mathrm{C}$ for $20 \mathrm{~min}$ in the dark. The cells were then washed twice with PBS, $200 \mu 1$ cell suspension $\left(1 \times 10^{6}\right.$ cells $\left./ \mathrm{ml}\right)$ was added to the 96-well black plate and fluorescence units were detected with an Infinite M200 PRO plate reader (Tecan Group, Ltd.) at 510/580 nm excitation/emission wavelengths.

Construction of the overexpression vectors and transfection. According to the PDB ID of ETC complexes I, III and V in molecular docking, the representative subunits of corresponding human genes were selected as NADH-ubiquinone oxidoreductase core subunit S1(NDUFS1), biquinol-cytochrome $c$ reductase core protein 1 (UQCRC1) and ATP synthase F1 subunit beta (ATP5F1B), respectively. The NDUFS1, UQCRC1 and ATP5F1B cDNA fragments were 

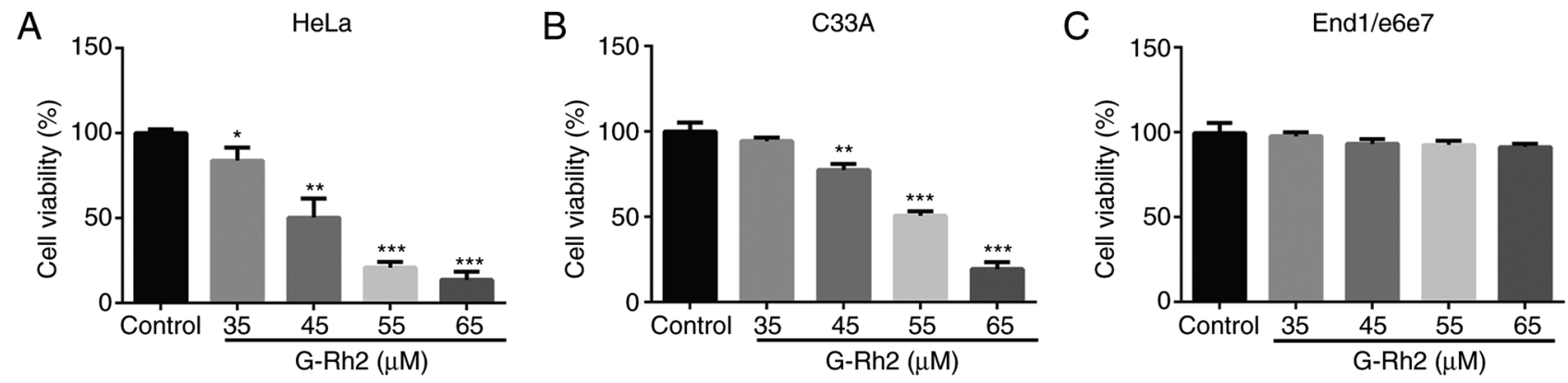

Figure 1. G-Rh2 reduces cervical cancer cell viability but has no cytotoxic effect on End1/e6e7 cells. Viability of cervical cancer cell lines (A) HeLa and (B) C33A and of (C) End1/e6e7 cells (control) following exposure to G-Rh2 (35, 45, 55 and $65 \mu \mathrm{M}$ ) for 24 h. Cell Counting Kit-8 assays were performed to measure cell viability, which was compared with that of the control group under $0.12 \% \mathrm{v} / \mathrm{v}$ DMSO. All results were obtained from three independent experiments $(\mathrm{n}=3)$. Data are presented as the mean \pm standard deviation and were analyzed by one-way ANOVA followed by Dunnet's post-hoc test. " $\mathrm{P}<0.05$; ${ }^{* *} \mathrm{P}<0.01 ;{ }^{* * *} \mathrm{P}<0.001$ vs. control. G-Rh2, ginsenoside Rh2.

amplified with primers using a cDNA library prepared from HeLa cells as the template. The PCR program was as follows: $98^{\circ} \mathrm{C}$ for $10 \mathrm{sec}, 58^{\circ} \mathrm{C}$ for $10 \mathrm{sec}$ and then $72^{\circ} \mathrm{C}$ for $1 \mathrm{~min}$ per $\mathrm{kb}$ for a total of 35 cycles. The target fragments were digested with double restriction enzymes and then ligated to the corresponding plasmids (Guangzhou RiboBio Co., Ltd.) with T4 Ligase (Takara Bio, Inc.) and then transformed into E. coli turbo (New England Biolabs). Positive recombinant plasmids were identified by restriction digestion and sequencing. All plasmids, primers and enzymes used are provided in Table SI. For transfection, HeLa cells were seeded in 6-well plates and cultured for $24 \mathrm{~h}$. Cells were transfected with $2 \mu \mathrm{g}$ of the plasmids using Lipofectamine ${ }^{\circledR} 2000$ reagent (Invitrogen; Thermo Fisher Scientific, Inc.) for 6 h. The culture medium was then replaced with fresh opti-DMEM (Gibco; Thermo Fisher Scientific, Inc.) and the cells were continued to be grown in culture for $48 \mathrm{~h}$. For G-Rh 2 treatment, HeLa cells were treated with $45 \mu \mathrm{M} \mathrm{G}-\mathrm{Rh} 2$ for $24 \mathrm{~h}$ after transfection.

Statistical analysis. Statistical analyses were performed with GraphPad Prism software version 6 (GraphPad Software Inc.). For quantitative analyses, data were collected from three independent experiments and presented as the mean \pm standard deviation. One-way ANOVA followed by Dunnett's post-hoc test or Tukey's post-hoc test as indicated within the figure legends were utilized to compare among groups. $\mathrm{P}<0.05$ was considered to indicate a statistically significant difference.

\section{Results}

G-Rh2 inhibits the viability of cervical cancer cells but is not cytotoxic to Endl/e6e 7 cells. To study the impact of G-Rh2 on the viability of cancer and normal cells, two cervical cancer cell lines, including HeLa (integrated HPV18) and C33A (without HPV) and human cervical epithelial cells (End1/e6e7) as a control were exposed to $35,45,55$ and $65 \mu \mathrm{M} \mathrm{G}-\mathrm{Rh} 2$ for $24 \mathrm{~h}$. As presented in Fig. 1A and B, G-Rh2 was cytotoxic to all cervical cancer cell lines assessed, with IC50 values of $45 \mu \mathrm{M}$ for HeLa and $55 \mu \mathrm{M}$ for C33A cells at $24 \mathrm{~h}$. However, compared with the effect on the two cervical cancer cell lines, the effect of treatments at all concentrations on the viability of End1/e6e7 cells was negligible, indicating that G-Rh2 was highly selective for cancer cells (Fig. 1C).
G-Rh2 induces apoptosis in cervical cancer cells. Next, Annexin V-FITC/PI and Hoechst 33342 staining assays were used to analyze the number of apoptotic cells following exposure to different concentrations of G-Rh2. As indicated in Fig. 2A, after $24 \mathrm{~h}$ of treatment, the rate of early apoptosis was considerably increased in G-Rh2-treated cervical cancer cells (HeLa and C33A) compared with that in the control group. Furthermore, more intense blue staining and more irregular chromatin were observed in G-Rh2-treated HeLa and C33A cells (Fig. 2B) compared with that in the control group, indicating that G-Rh2 treatment induced apoptosis in cervical cancer cells. Of note, G-Rh2 significantly induced early apoptosis in both cancer cell lines in a dose-dependent manner. However, treatment with high-dose G-Rh2 (55 and $65 \mu \mathrm{M})$ slightly increased the rate of cell death in End1/e6e7 cells compared with that in the control group (Fig. S1) and demonstrated that the effect on normal cells was not statistically significant as determined for cancer cells.

HeLa cells are more sensitive to G-Rh2 than C33A cells regarding $M M P$ and ATP generation. According to a previous study by our group suggesting that G-Rh2 possibly induced mitochondrial dysfunction in cancer cells, the mitochondrial defects induced by G-Rh2 may potentially result in apoptosis of cervical cancer cells (24). In the present study, to further examine this, the JC-1 and rhodamine 123 probes were used to detect changes in the MMP, an index of mitochondrial function. After staining with the JC-1 fluorescent probe, HeLa cells had an obvious response, displaying red to green transition with increasing G-Rh2 concentration. However, only a slight change in JC-1 probe fluorescence was identified in C33A cells (Fig. 3A). In addition, flow cytometry was employed to measure the fluorescence intensity of rhodamine 123; G-Rh2 treatment significantly reduced the MMP in HeLa cells. Although $55 \mu \mathrm{M}$ G-Rh2 was able to significantly reduce the MMP in C33A cells, the degree of reduction was significantly lower than that in HeLa cells (Fig. 3B). Next, the effect of G-Rh2 on cellular ATP levels was evaluated by a chemiluminescence assay. Specifically, G-Rh2 treatment reduced cellular ATP levels by $10.4 \%(35 \mu \mathrm{M})$ and $49.1 \%$ $(45 \mu \mathrm{M})$ in HeLa cells and by $7.5 \%(35 \mu \mathrm{M})$ and $35.2 \%$ $(45 \mu \mathrm{M})$ in C33A cells compared with that in in the control group (Fig. 3C). According to these observations, HeLa cells 
A

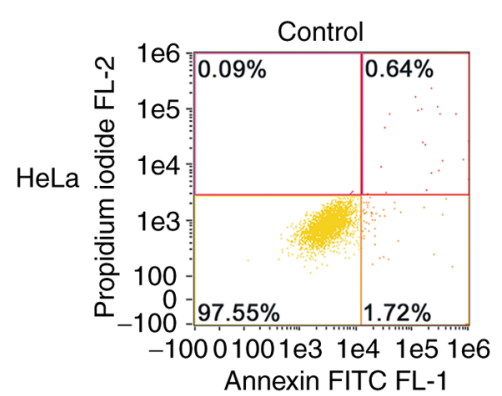

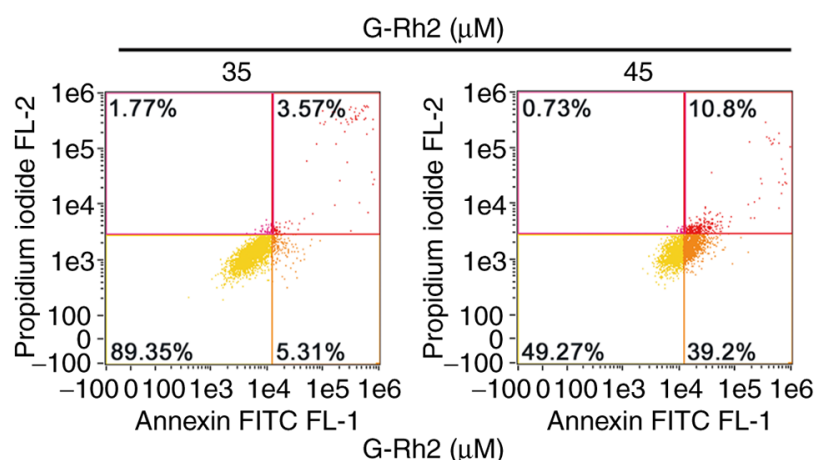
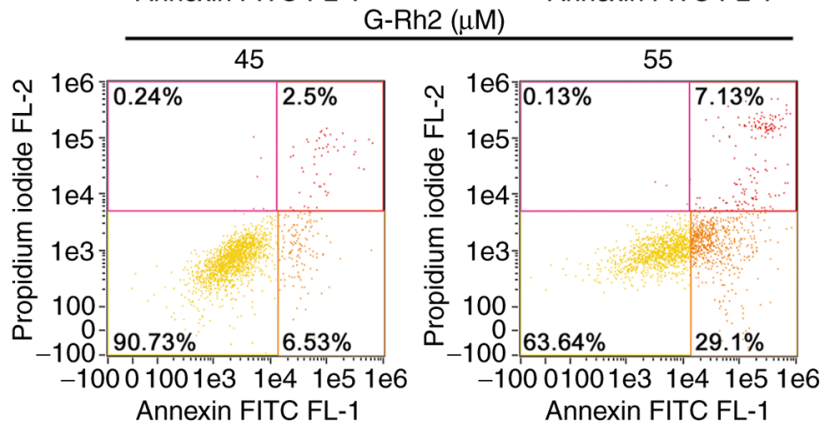
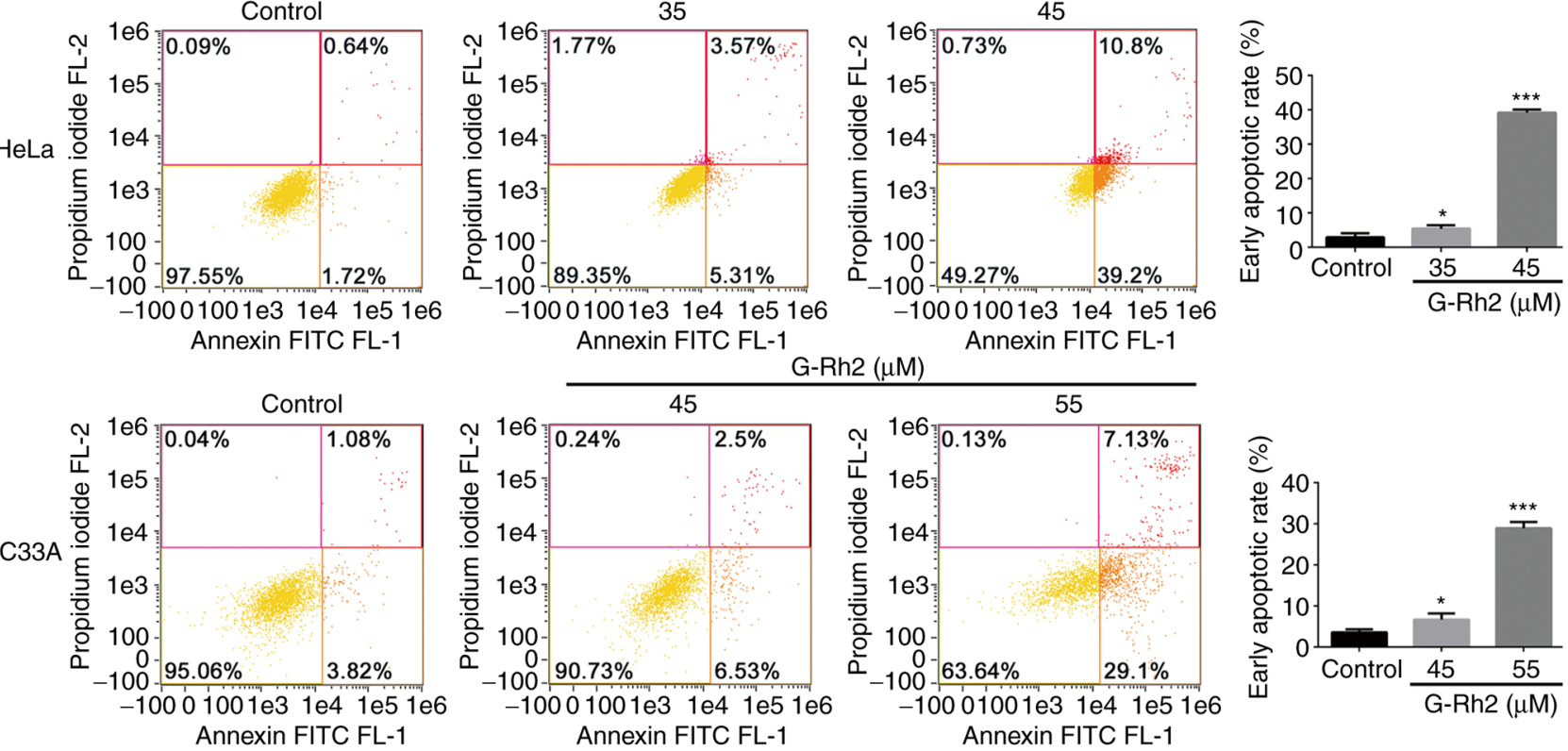

B
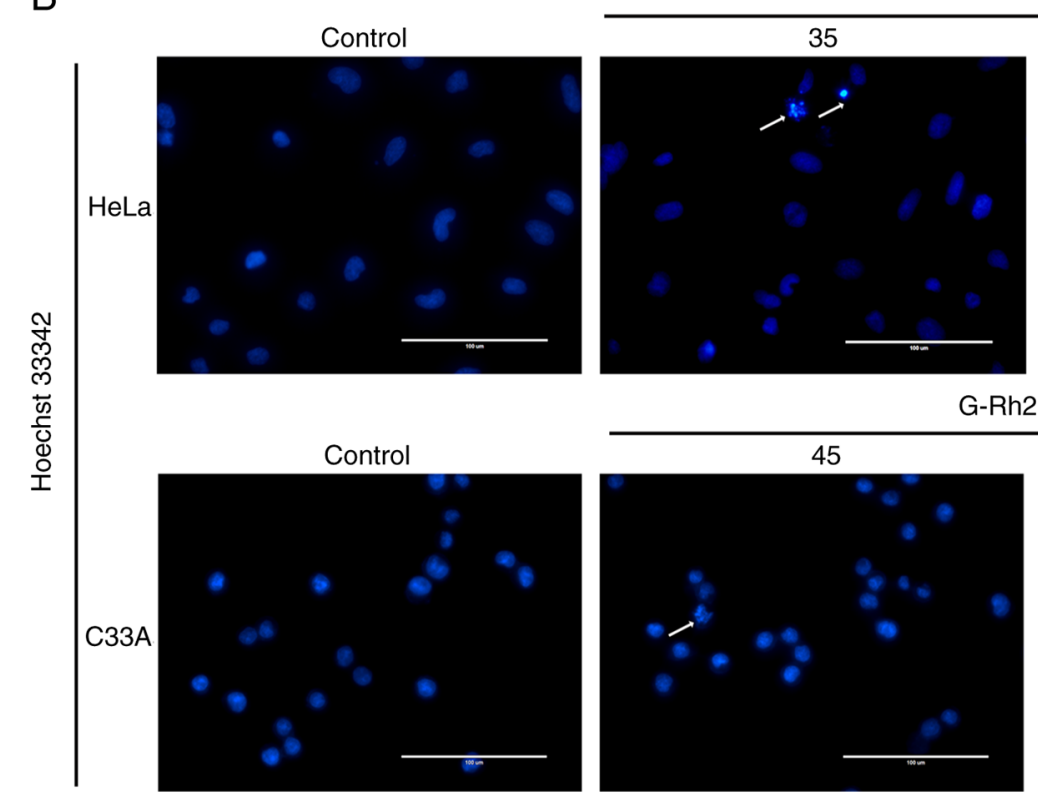

G-Rh2 $(\mu \mathrm{M})$

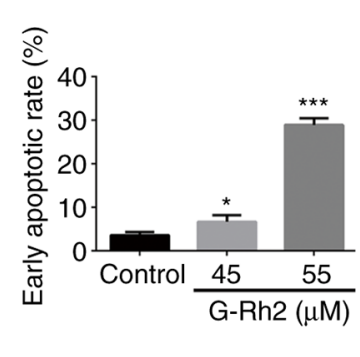

G-Rh2 $(\mu \mathrm{M})$
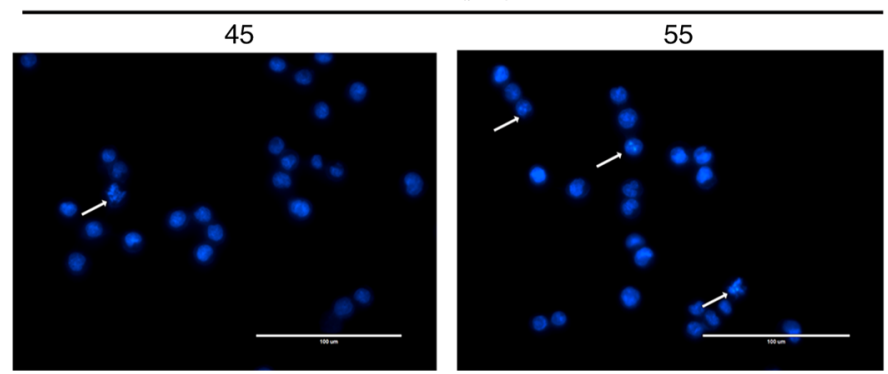

Figure 2. Treatment with G-Rh2 promotes apoptosis in cervical cancer cells. (A) Representative flow cytometry images indicating apoptosis of HeLa and C33A cells after G-Rh2 treatment. The early apoptotic rate was quantified by IDEAS software v6.1. (B) Representative image of Hoechst staining indicating the morphological changes to apoptotic HeLa and C33A cells following G-Rh2 treatment (scale bar, $100 \mu \mathrm{m}$ ). White arrows represent nuclei with intense fluorescence and an irregular shapes. Data are presented as the mean \pm standard deviation of three independent experiments and were analyzed by one-way ANOVA followed by Dunnet's post-hoc test. ${ }^{*} \mathrm{P}<0.05 ;{ }^{* * *} \mathrm{P}<0.001$ vs. control. G-Rh2, ginsenoside Rh2.

were more sensitive to G-Rh2 than C33A cells, at least with regard to MMP and ATP production. Therefore, HeLa cells were selected for subsequent mechanistic examination of these effects.

Inhibitory effect of G-Rh2 on mitochondrial OXPHOS is greater than that on glycolysis in HeLa cells. OXPHOS is essential for the ability of cells to hold the MMP (7). To study the effect of G-Rh2 on mitochondrial OXPHOS, a Seahorse XFp extracellular flux analyzer was used to monitor mitochondrial aerobic respiration in HeLa cells. As presented in Fig. S2A,
G-Rh2 treatment of HeLa cells resulted in a rapid decline of OXPHOS. G-Rh2 treatment also significantly inhibited the cellular response to typical ETC complex inhibitors [e.g., oligomycin (ATP synthase inhibitor), FCCP (mitochondrial uncoupling agent), AA (ETC complex III inhibitor) and rot (ETC complex I inhibitor)]. In addition, G-Rh2 significantly inhibited the basal and maximum mitochondrial OXPHOS capacity. Furthermore, in HeLa cells, ATP-linked proton leak as well as reserve capacity were also more significantly inhibited by G-Rh2 compared with those in the control group (Fig. S2B). 
A
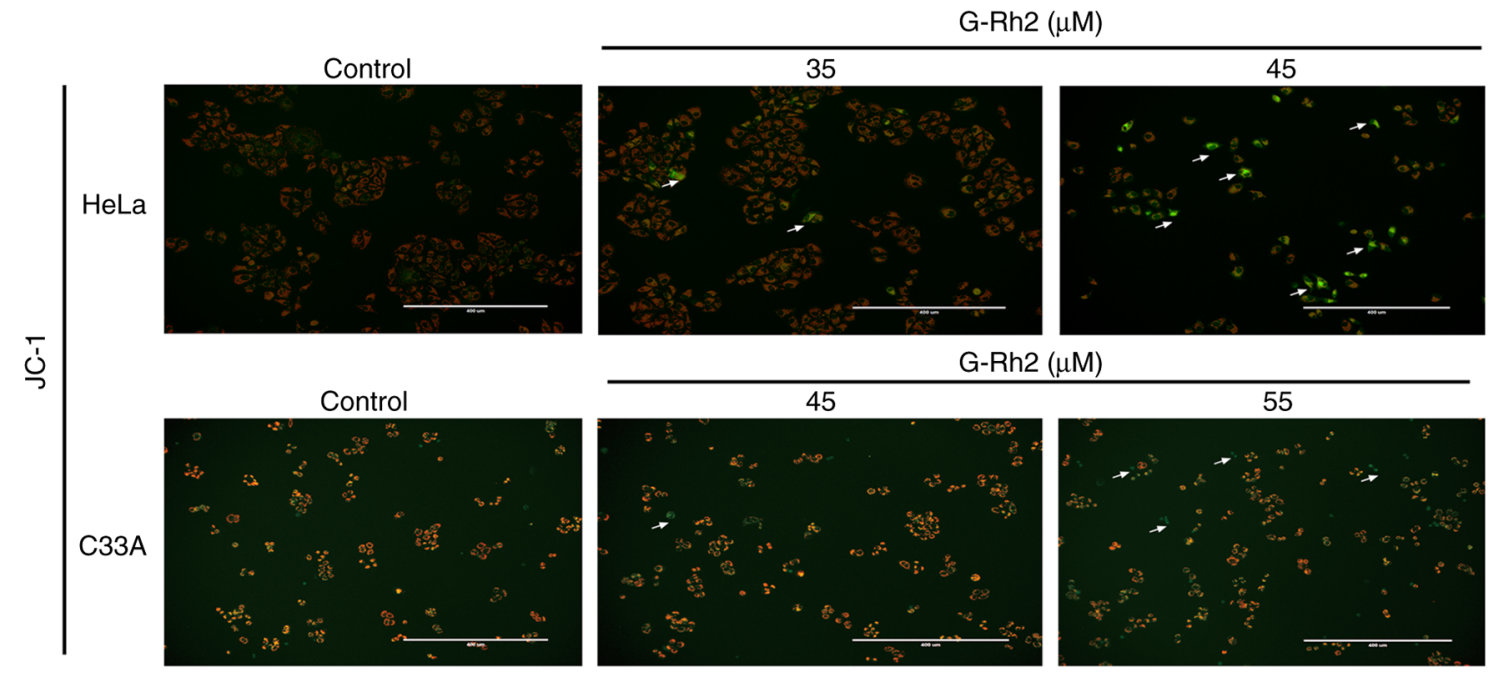

B
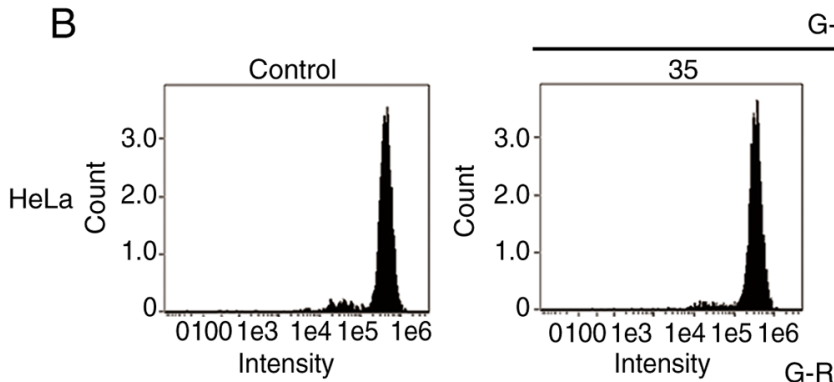

$\mathrm{G}-\mathrm{Rh} 2(\mu \mathrm{M})$
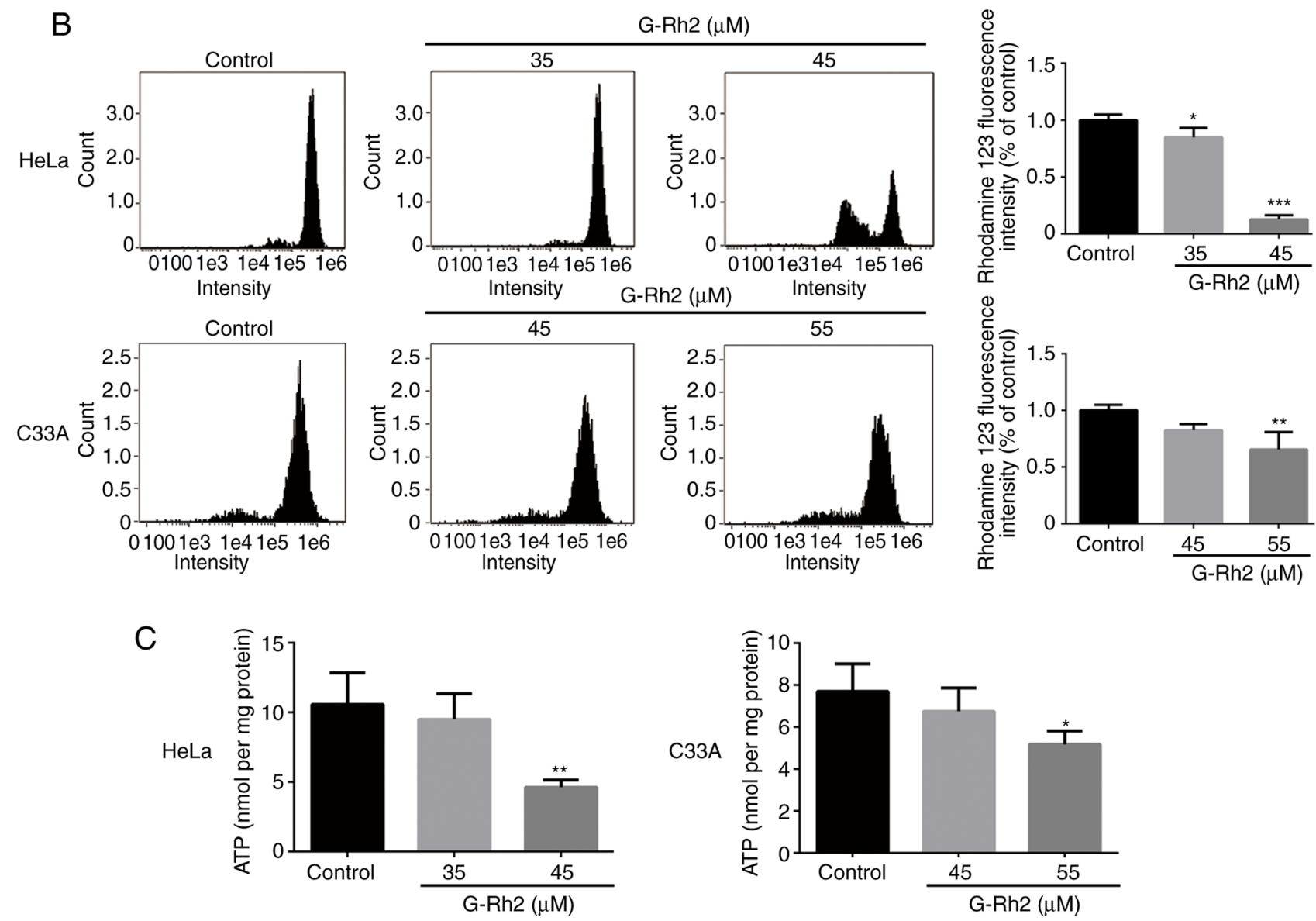

Figure 3. Greater inhibitory effect of G-Rh2 on MMP and ATP production in HeLa cells compared with that in C33A cells. (A) Representative image of MMP as measured by the JC-1 fluorescent probe in HeLa and C33A cells after G-Rh2 treatment (scale bar, $400 \mu \mathrm{m}$ ). The white arrows represent that the fluorescence color changes from red to green and the MMP decreases. (B) Representative flow cytometry histograms indicating the Rhodamine 123 fluorescence intensity of HeLa and C33A cells after G-Rh2 treatment and quantification by IDEAS software v6.1. (C) Changes in intracellular ATP levels in HeLa and C33A cells following exposure to G-Rh2. Data are presented as mean \pm standard deviation of three independent experiments and were analyzed by one-way ANOVA followed by Dunnet's post-hoc test. ${ }^{*} \mathrm{P}<0.05 ;{ }^{* *} \mathrm{P}<0.01 ;{ }^{* * * *} \mathrm{P}<0.001$ vs. control. G-Rh2, ginsenoside Rh2; MMP, mitochondrial membrane potential.

Cancer cells provide energy and produce lactic acid through the glycolysis pathway, via a process termed the 'Warburg effect'. Therefore, it was investigated whether G-Rh2 was involved in blocking glycolysis. The results indicated that G-Rh2 reduced ECAR, which reflects glycolytic capacity. Glycolysis, glycolytic capacity and glycolytic reserve were all significantly inhibited by G-Rh2 (Fig. S2C and D). Of note,
G-Rh2 inhibited both OXPHOS and glycolysis in HeLa cells and had a stronger effect on OXPHOS.

Impairment of OXPHOS by G-Rh2 occurs via direct inhibition of ETC complexes activity, not by downregulation of ETC proteins. Decreasing OXPHOS may contribute to the downregulation of ETC proteins or to the inhibition of 
A

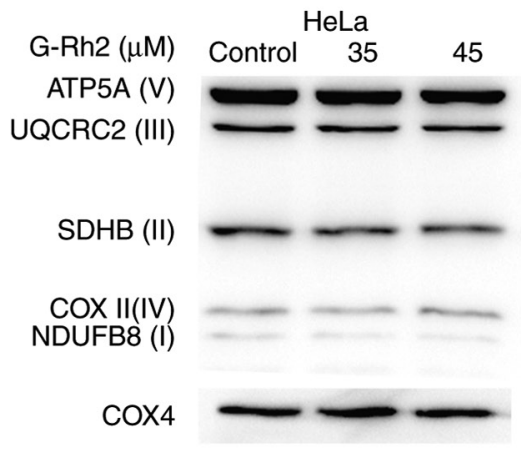

B

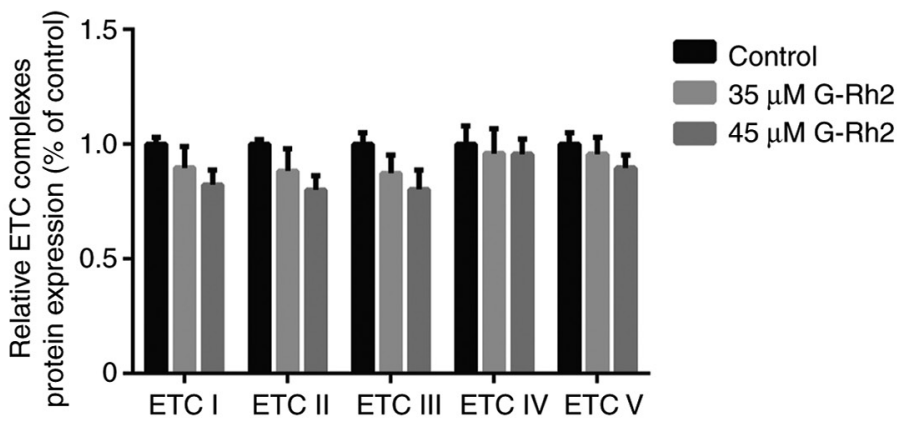

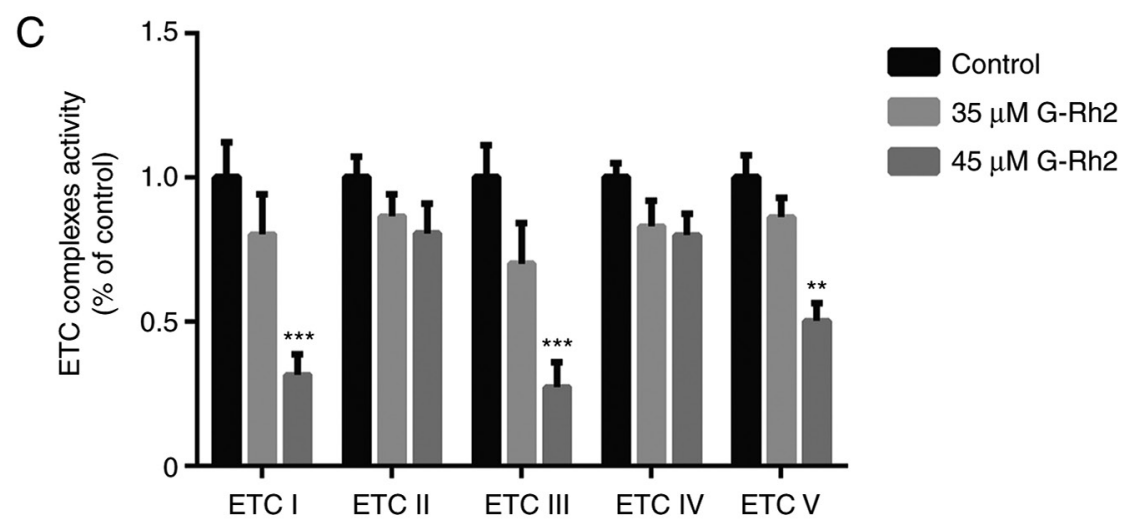

Figure 4. G-Rh2 decreases oxidative phosphorylation by directly inhibiting the activity of ETC complexes instead of regulating their protein expression. (A) Representative western blot image indicating ETC protein expression after G-Rh2 treatment. COX4 was used as the sample loading control. (B) Quantitative expression of ETC protein complexes. (C) Activities of ETC complexes I-V after G-Rh2 treatment compared with those in the control group. Quantitative bar graphs were generated separately and then plotted together. Data are presented as the mean \pm standard deviation of three independent experiments and were analyzed by one-way ANOVA followed by Dunnet's post-hoc test. ${ }^{* *} \mathrm{P}<0.01 ;{ }^{* * *} \mathrm{P}<0.001$ vs. control. G-Rh2, ginsenoside Rh2; ETC, electron transport chain; NDUFB8, NADH-ubiquinone oxidoreductase subunit B8; SDHB, succinate dehydrogenase complex iron sulfur subunit B; UQCRC2, ubiquinol-cytochrome $\mathrm{C}$ reductase core protein 2; COX II, cytochrome oxidase subunit 2; ATP5A, adenosine triphosphate 5A.

ETC complex enzymes (31). Therefore, in the present study, the expression levels of five ETC subunits were detected in G-Rh2-treated cells. As presented in Fig. 4A and B, ETC protein expression was not significantly altered after G-Rh2 treatment, indicating that G-Rh2 damages OXPHOS not by downregulating ETC protein expression but by directly inhibiting the activity of ETC complexes. As expected, treatment with $45 \mu \mathrm{M}$ G-Rh2 significantly inhibited the activity of ETC complexes I, III and V in HeLa cells but did not affect ETC complexes II and IV (Fig. 4C). These results suggested that the impairment of G-Rh2 on OXPHOS was not caused by downregulation of ETC protein expression but by directly inhibiting the activity of ETC complexes.

Increased ROS levels in G-Rh2-mediated apoptosis. MtROS is an outgrowth of OXPHOS that is primarily generated by the ETC complexes. As G-Rh2 significantly inhibited the activity of complexes I, III and V, its impact on mtROS production was examined. As presented in Fig. 5A, treatment of HeLa cells with $45 \mu \mathrm{M}$ G-Rh2 led to overproduction of mtROS, with mtROS levels exhibiting a significant increase of 2.8-fold compared with that in the control group. This burst of mtROS production was partially rescued by the antioxidant mitoQ.

To study whether mtROS was involved in G-Rh2-induced apoptosis, the protective effect of mitoQ on G-Rh2-induced cytotoxicity was detected using CCK-8 assays. As indicated in Fig. 5B, the effect of G-Rh2 on HeLa cells was partially attenuated by mitoQ pretreatment. Furthermore, quantitative measurements of Annexin V/PI-positive cells by flow cytometry (Fig. 5C) verified that mitoQ partially rescued G-Rh2-induced early apoptosis. These results demonstrated that G-Rh2-induced mtROS generation mediated the subsequent apoptosis.

Molecular docking reveals a putative $G-R h 2$ binding site on ETC complexes I, III and V. Although the above results confirmed that G-Rh2 induced $\mathrm{mtROS}$, triggering apoptosis via suppressing ETC complexes I, III and V, the detailed binding mechanism remained to be further elucidated. Therefore, protein-ligand docking was performed to understand the mode of G-Rh2 binding and to locate the ETC complex residues that may have crucial roles in G-Rh2 binding.

The molecular docking results revealed that the optimal docking conformation binding energy of G-Rh2 and ETC complex III was $-10.6 \mathrm{kcal} / \mathrm{mol}$, which was lower than that of ETC complex I $(-7.73 \mathrm{kcal} / \mathrm{mol})$ and ETC complex V (-6.48 $\mathrm{kcal} / \mathrm{mol})$. The hydroxyl oxygen atom of the six-membered ring of G-Rh2 was indicated to be able to form hydrogen bonds with Thr87 in ETC complex I, Ile122 in ETC complex III and Thr163 and Asp256 in ETC complex V, with hydrogen bond lengths of $3.3,2.1,1.9$ and $3.1 \AA$, respectively. The oxygen atom of the six-membered ring of G-Rh2 was also able to 
A

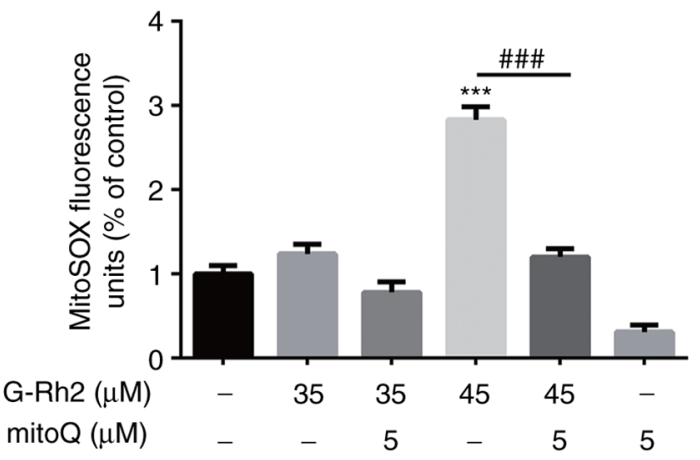

B

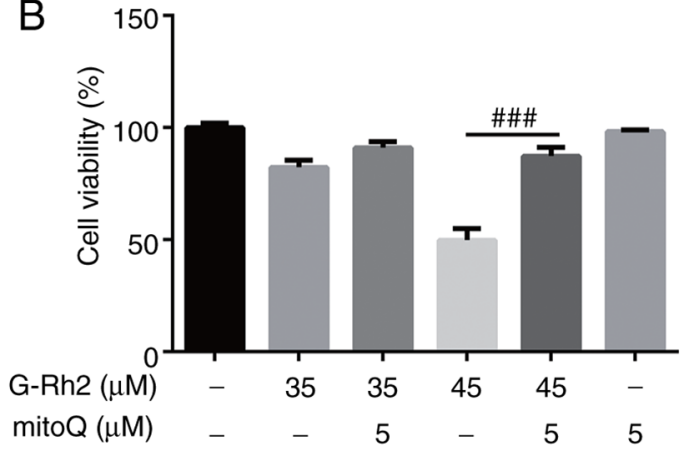

C
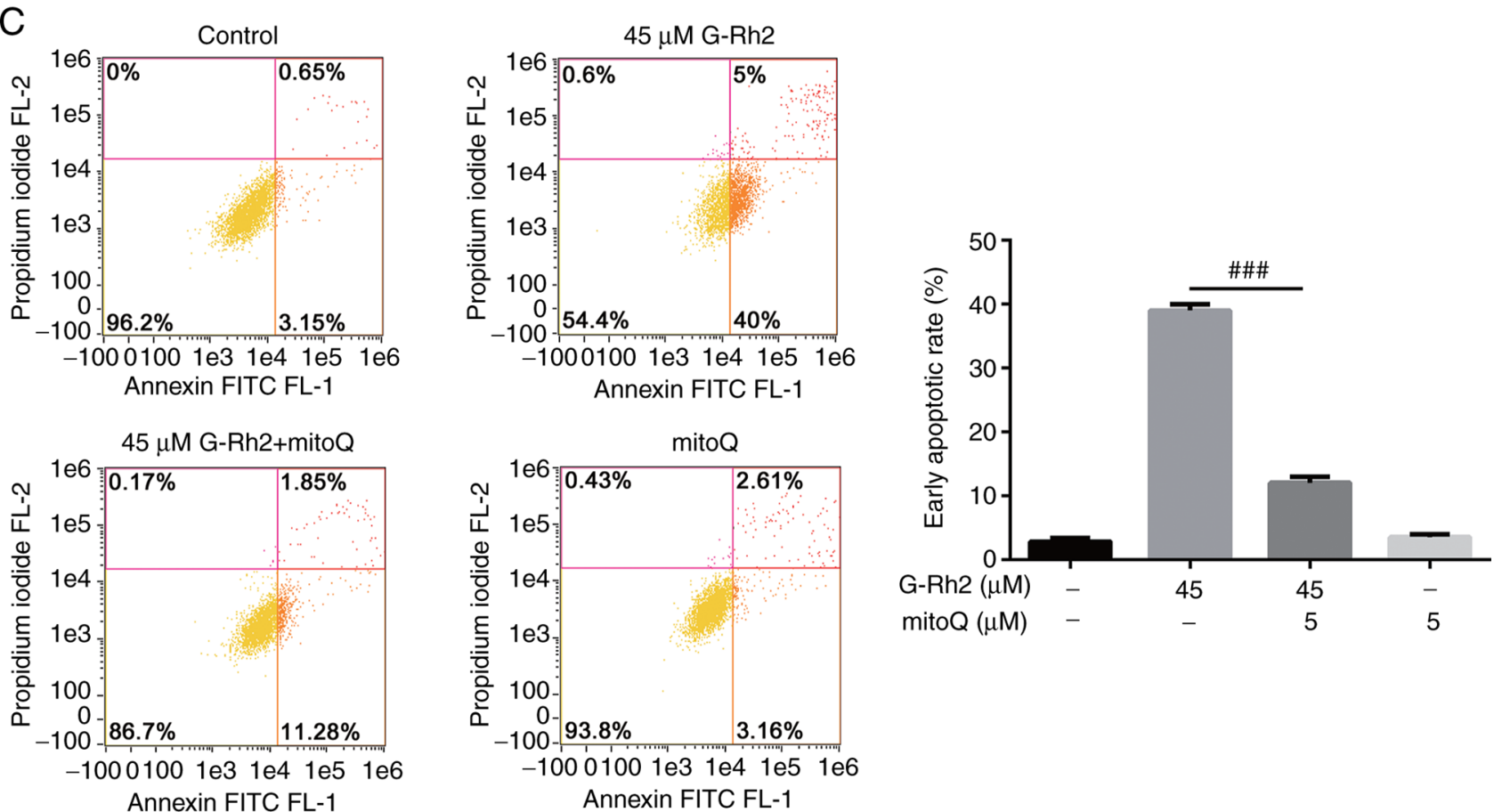

Figure 5. Apoptosis induced by G-Rh2 is related to mtROS. HeLa cells were pretreated with or without mitoQ ( $5 \mu \mathrm{M})$ for $1 \mathrm{~h}$ and then exposed to the indicated concentrations of G-Rh2 for 24 h. (A) The mitoSOX fluorescence units in HeLa cells were detected using the Infinite M200 PRO plate reader. Data were analyzed by one-way ANOVA followed by Tukey's post-hoc test and are presented as the mean \pm standard deviation of three independent experiments. (B) Cell viability was detected using Cell Counting Kit-8 assays. (C) Annexin V-FITC/propidium iodide staining was applied to measure the percentage of apoptotic cells, which was quantified by flow cytometry. Data are presented as the mean \pm standard deviation of three independent experiments and were analyzed by one-way ANOVA followed by Tukey's post-hoc test in A or Dunnet's post-hoc test in B and C. ${ }^{* * *} \mathrm{P}<0.001$ vs. without G-Rh2 and Mito Q; ${ }^{\# \#} \mathrm{P}<0.001$ vs. $45 \mu \mathrm{M}$ G-Rh2 without mitoQ. G-Rh2, ginsenoside Rh2.

form a $2.5 \AA$ hydrogen bond with Arg189 in ETC complex V. In addition, the oxygen of Tyr345 in ETC complex V formed a hydrogen bond with the six-membered ring hydrogen of G-Rh2, the length of which was $1.9 \AA$ (Fig. 6A and B; Table I). The formation of these hydrogen bonds enhanced the ability of G-Rh2 to target mitochondrial ETC complexes by better inhibiting protein activity.

Overexpression of UQCRC1 partially rescued G-Rh2-induced $m t R O S$ production and apoptosis. In order to verify that the effect of ETC complexes I, III and V in G-Rh2 induced cervical cancer cell apoptosis, overexpression vectors for the representative subunits of ETC complex I (NDUFS1), ETC complex III (UQCRC1) and ETC complex V (ATP5F1B) were constructed. HeLa cells transfected with these overexpression vectors exhibited a significant increase in the expression levels of NDUFS1, UQCRC1 and ATP5F1B protein compared to mock-transfected cells (Fig. 7A). G-Rh2 treatment increased mtROS and apoptosis in HeLa cells compared with that in the control group, which was partially attenuated by overexpression of NDUFS1, UQCRC1 and ATP5F1B and the overexpression of UQCRC1 had a significant effect (Fig. 7B and C).

\section{Discussion}

In the present study, the cytotoxicity of G-Rh2 in the HeLa (HPV18-positive) and C33A (HPV-negative) cervical cancer cell lines compared with that of the non-tumorigenic spontaneous End1/e6e7 cell line was assessed. The results demonstrated that G-Rh2 exposure had specific dose-dependent cytotoxicity on both cervical cancer cell lines but not on End1/e6e7 cells. It was further reported that G-Rh2-induced apoptosis was closely related to the specific activity inhibition of ETC complexes I, III and V, which led to reduced OXPHOS, 
Table I. Binding affinities (free energies of binding) and the types of interactions of the ligand ginsenoside Rh2 with its receptors ETC complex I, III and V.

\begin{tabular}{|c|c|c|c|}
\hline ETC complex & $\begin{array}{l}\text { Docking score } \\
(\mathrm{kcal} / \mathrm{M})\end{array}$ & $\begin{array}{l}\text { Residues involved in } \\
\text { hydrogen bonding }\end{array}$ & $\begin{array}{l}\text { Residues involved in } \\
\text { weak interactions }\end{array}$ \\
\hline I & -7.73 & THR87 & $\begin{array}{l}\text { PRO84, ASP85, SER86, THR87, VAL112, } \\
\text { ASN113, GLU126, GLU213, PHE258, } \\
\text { PHE509, PHE510, LYS511, GLY512 }\end{array}$ \\
\hline III & -10.60 & ILE122 & $\begin{array}{l}\text { ILE125, PHE129, TYR132, MET139, } \\
\text { ILE147, PRO271, LEU275, TYR279, } \\
\text { PHE278, LEU282, MET295, } \\
\text { PHE9, ILE299 }\end{array}$ \\
\hline V & -6.48 & $\begin{array}{l}\text { THR163, ASP256, } \\
\text { ARG189, TYR345 }\end{array}$ & $\begin{array}{l}\text { THR163, VAL164, ARG189, GLU192, } \\
\text { ASP256, TYR345, PHE424, ALA421, } \\
\text { THR425 }\end{array}$ \\
\hline
\end{tabular}

ETC, electron transport chain.

A

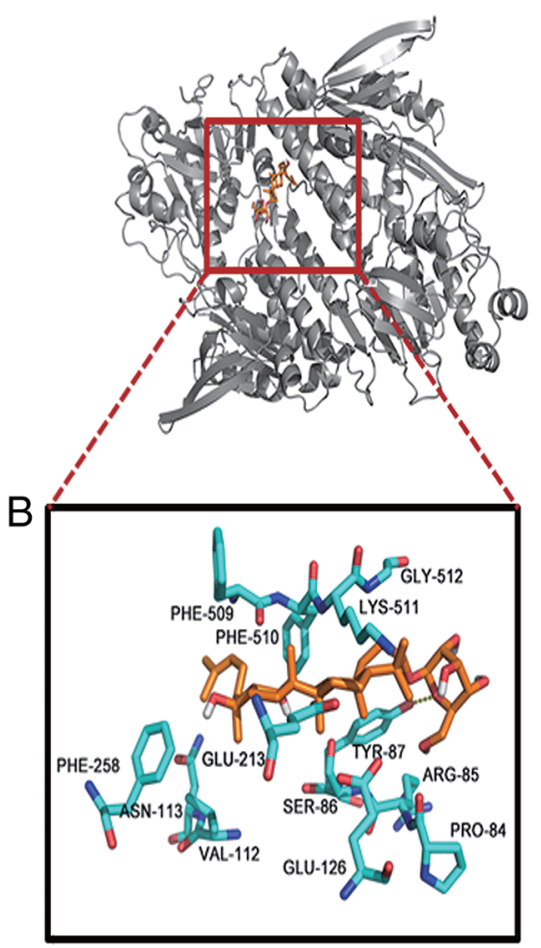

ETC complex III

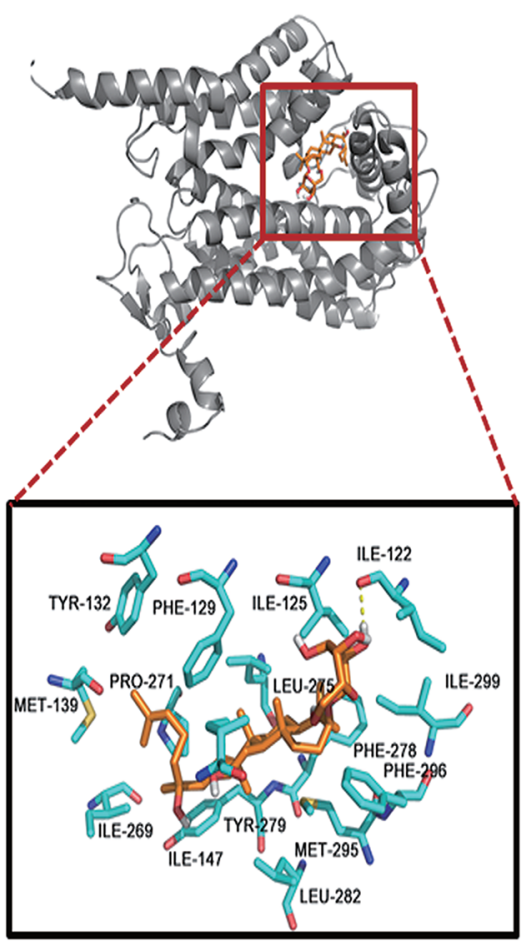

ETC complex V

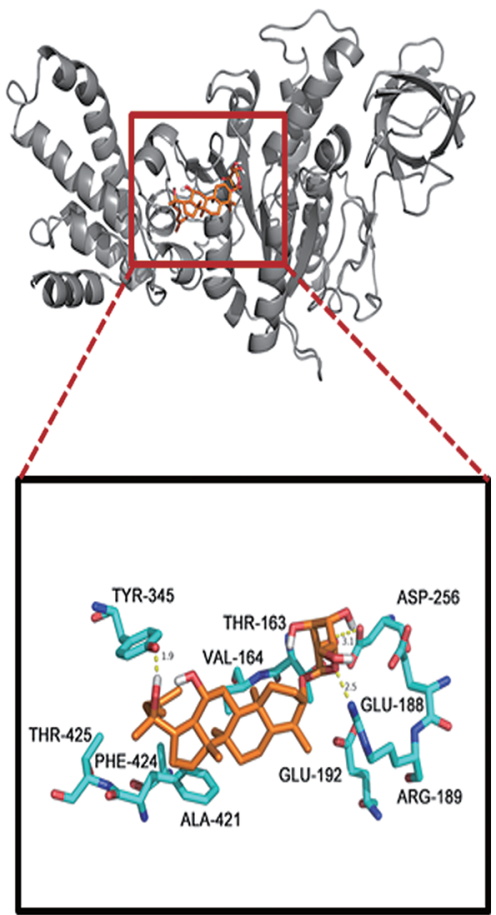

Figure 6. Binding of G-Rh2 to ETC complexes I/III/V. (A) Schematic diagram of G-Rh2 ligand binding to protein components of ETC complex I, III and V. The proteins are displayed in cartoon format. (B) Schematic diagram of the amino acid site of G-Rh2 ligand binding to ETC complex I, III and V proteins. G-Rh2, ginsenoside Rh2; ETC, electron transport chain.

decreased oxygen consumption and increased mtROS production; these processes are summarized in Fig. 8.

The vast majority of G-Rh2-based anti-cervical cancer studies have been performed in HeLa cells $(32,33)$. The present results are consistent with these reports; in addition, it was indicated that G-Rh2 was cytotoxic to both HeLa and C33A cells, with IC50 values of 45 and $55 \mu \mathrm{M}$, respectively. Compared with the effect in C33A cells, HeLa cells had a more markedly decreased viability following treatment with a relatively low concentration of G-Rh2. This may indicate that cervical cancer caused by HPV infection is more sensitive to G-Rh2. By contrast, G-Rh2 did not inhibit the viability of End1/e6e7 cells to a marked degree, although their viability was slightly decreased at higher concentrations tested. Similar to reports on other human cancer cell lines $(34,35)$, the specific effect of G-Rh2 on cervical cancer cells determined in the present study suggests its low intrinsic toxicity and its differential effects on normal cells vs. cancer cells. 
A

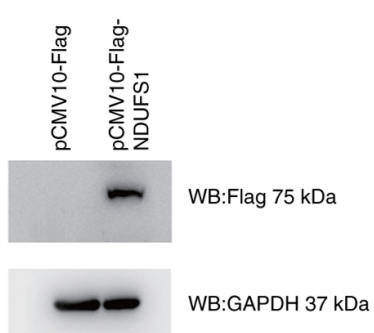

B

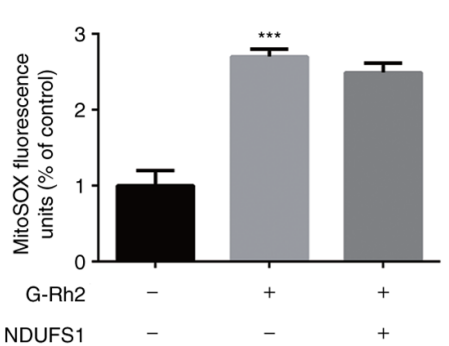

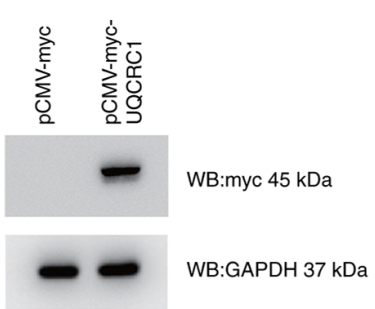

UQCRC1

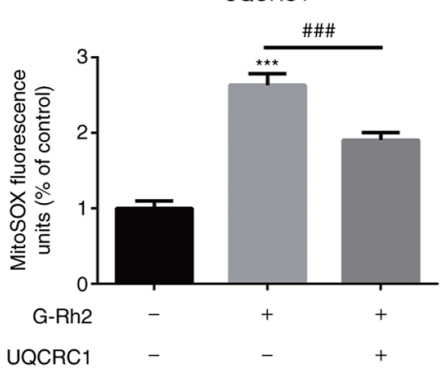

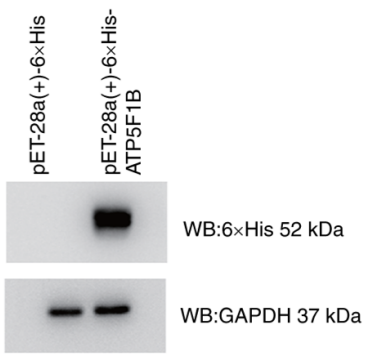

ATP5F1B

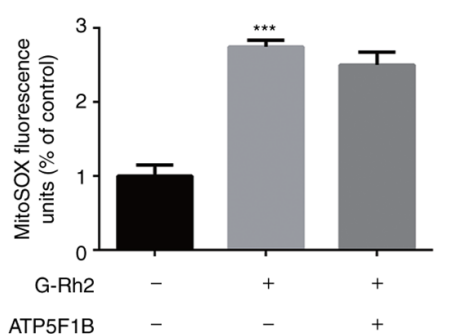

C
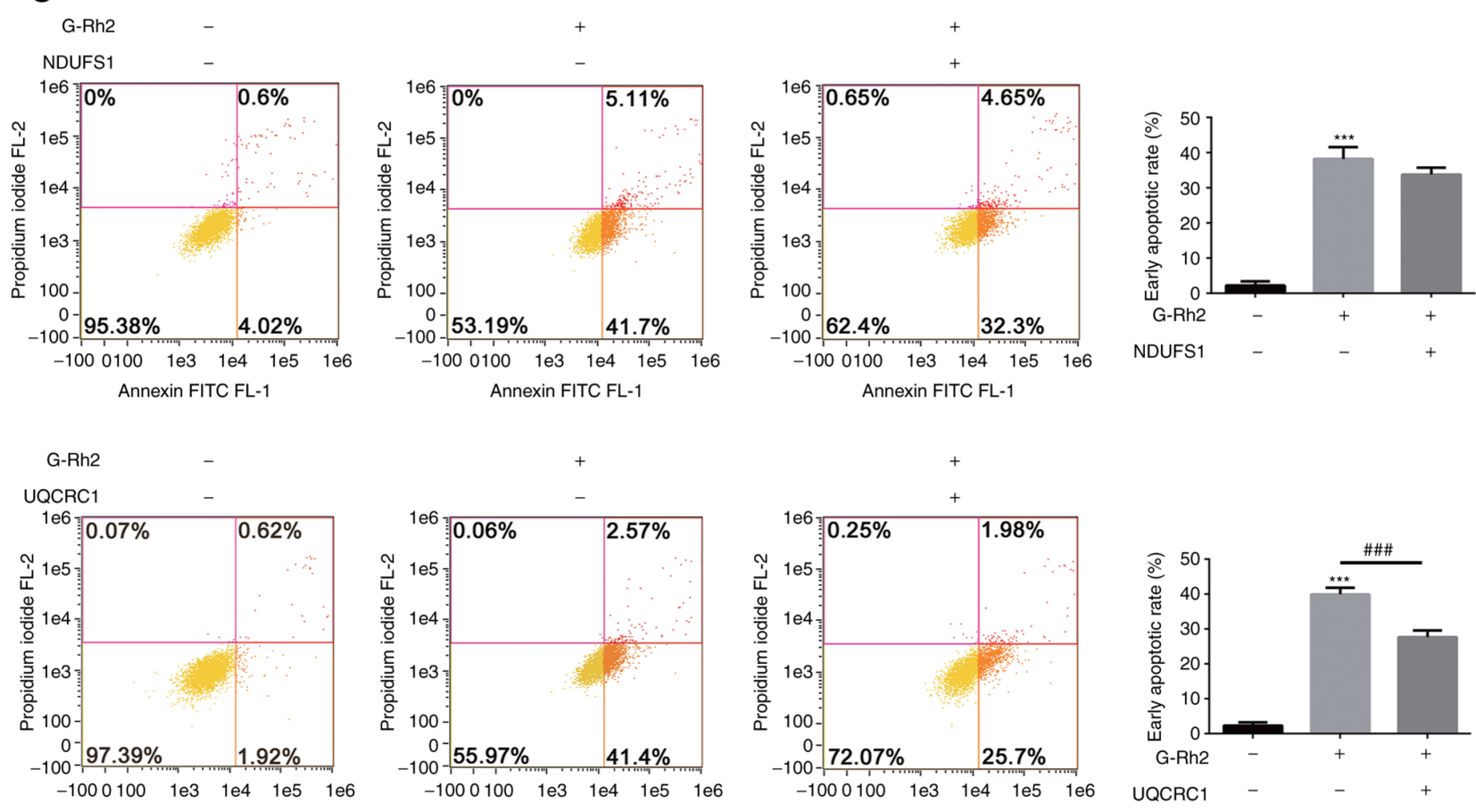

Annexin FITC FL-1
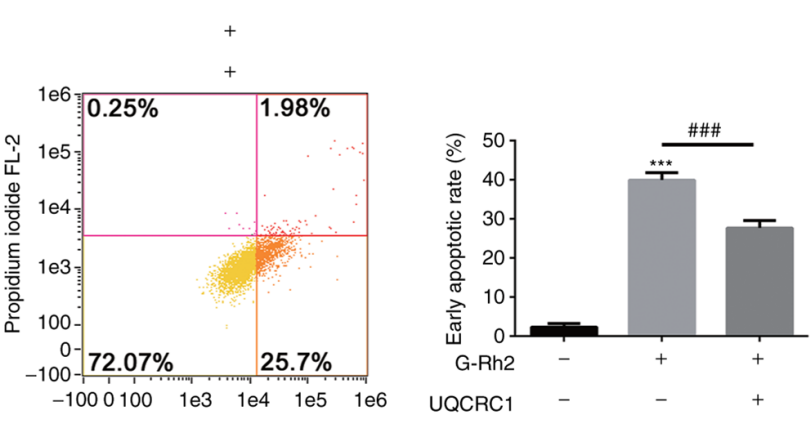

Annexin FITC FL-1

Annexin FITC FL-1

Annexin FITC FL-1
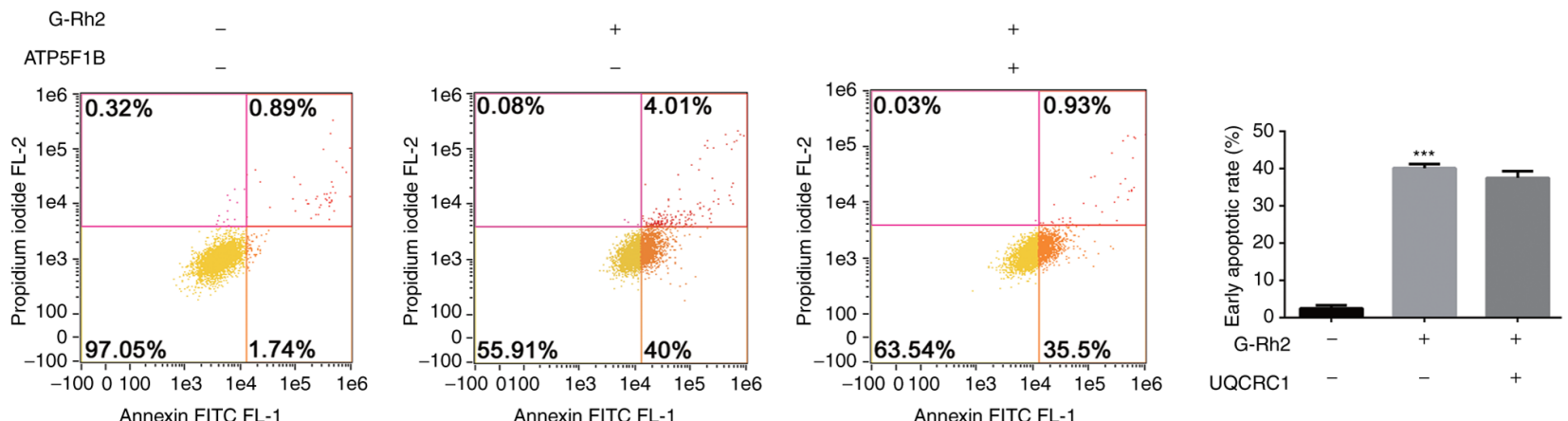

Figure 7. Overexpression of UQCRC1 partly rescued G-Rh2-induced mtROS and apoptosis. (A) Total protein extracts from HeLa cells expressing flag-tagged NDUFS1, myc-tagged UQCRC1 and 6x-his-tagged ATP5F1B were used for WB analysis with an anti-flag/myc/6x-his-tag antibody. GAPDH was used as the sample loading control. (B) The mitoSOX fluorescence units in HeLa cells were recorded using an Infinite M200 PRO plate reader. (C) Flow cytometry was used to detect the percentage of apoptotic cells, which was quantified by IDEAS software v6.1. Data are presented as the mean \pm standard deviation of three independent experiments and were analyzed by one-way ANOVA followed by Dunnet's post-hoc test. ${ }^{* * *} \mathrm{P}<0.001$ vs. mock-transfected group. ${ }^{\# \# \# ~} \mathrm{P}<0.001 \mathrm{vs}$. $45 \mu \mathrm{M}$ G-Rh2 without UQCRC1 overexpression. G-Rh2, ginsenoside Rh2; WB, western blot; NDUFS1, NADH dehydrogenase Fe-S protein 1; UQCRC1, ubiquinol-cytochrome c reductase core protein 1; ATP5F1B, ATP synthase F1 subunit beta. 


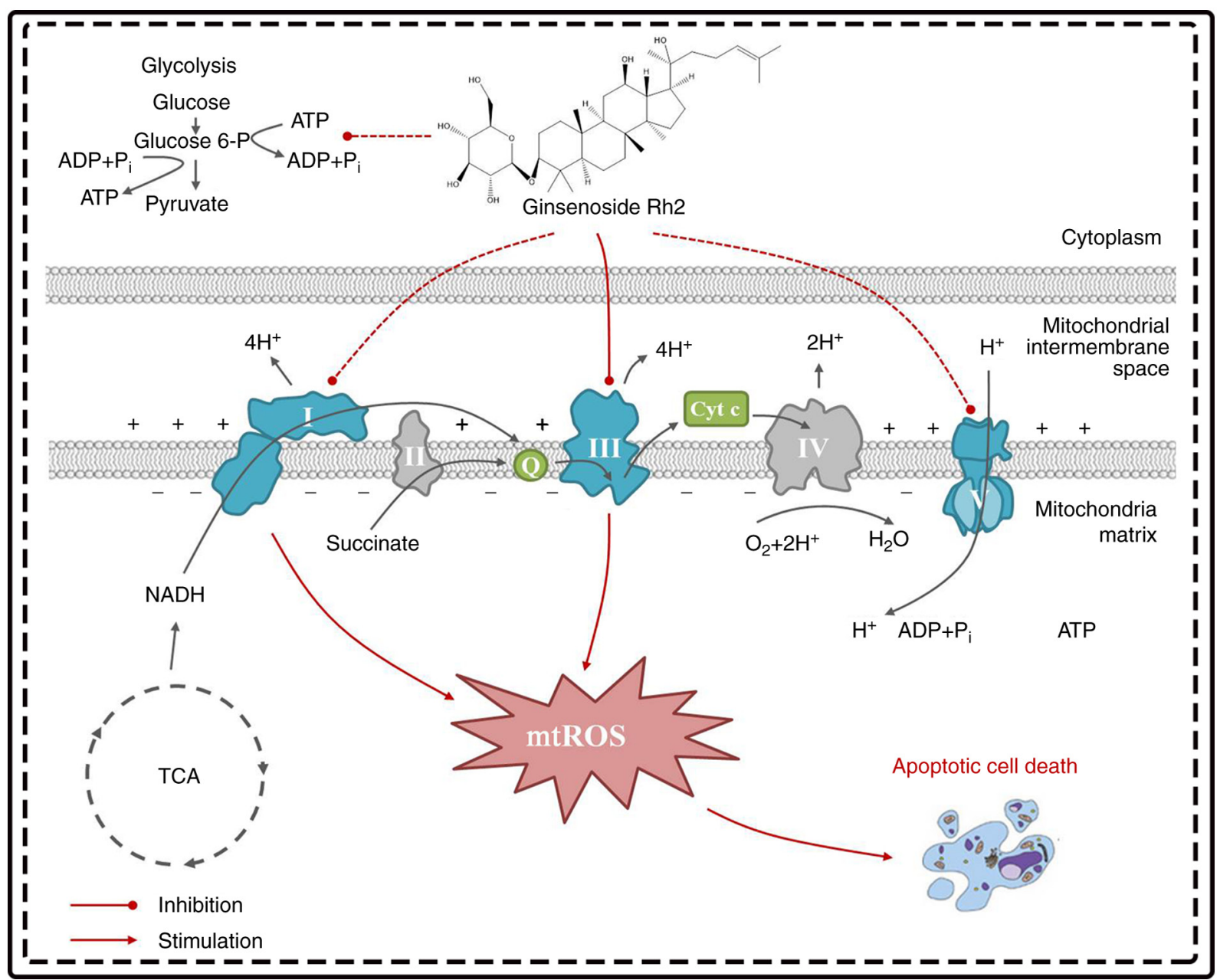

Figure 8. Schematic diagram of G-Rh2 targeting mitochondria. In the process of reducing mitochondrial metabolism, G-Rh2 primarily targets ETC complexes III and indirectly affects I and V, thereby impairing mitochondrial oxidative phosphorylation, blocking electron transfer related to the TCA cycle and stimulating excess mtROS production, leading to apoptosis. G-Rh2, ginsenoside Rh2; ETC, electron transport chain; mtROS, mitochondrial reactive oxygen species; Cyt c, cytochrome c; Q, ubiquinone; TCA, tricarboxylic acid.

One of the major apoptotic pathways in cancer cells involves the mitochondria. The presence of mitochondrial dysfunction was considered as a possible mechanism of G-Rh2-induced cytotoxicity (36). A previous study by our group suggested that G-Rh2 upregulated voltage-dependent anion channel 1 to trigger the mitochondrial translocation of BAX, promoting cytochrome c release and initiating mitochondrial-dependent apoptosis in HeLa cells (24). In the present study, it was revealed that the effect of G-Rh2 on the dissipation of the MMP and the reduction in ATP generation in HeLa cells was much stronger than that in C33A cells, consistent with the cell viability and apoptosis results. It is worth noting that p53 is mutated in C33A cells, while p53 is wild-type in HeLa (HPV-positive cervical cancer cell line) and p53 is involved in maintaining mitochondrial homeostasis. The response of wild-type p53 to mitochondrial stress in HeLa cells may be responsible for the higher sensitivity to decreased MMP and ATP levels $(37,38)$. In summary, mitochondria may be the primary intracellular target of G-Rh2.

Mitochondria primarily synthesize ATP via OXPHOS, which involves the ETC complex along with several carriers that localize to the inner mitochondrial membrane (39). The next goal of the present study was to investigate whether
G-Rh2 affected mitochondrial OXPHOS and the underlying mechanism. In these experiments, it was demonstrated that G-Rh2 inhibited mitochondrial respiration (basal OCR, maximal OCR and reserve capacity). Mitochondrial defects may lead to reduced expression of ETC complexes I-V (40). The results suggested that G-Rh2 primarily suppressed ETC by inhibiting the activity of ETC complex enzymes rather than by affecting their expression. It was further demonstrated that G-Rh2 exhibited potent inhibitory effects on ETC complexes I, III and V, but had no impact on ETC complexes II and IV. The suppression of ETC complexes I, III and V by G-Rh2 caused significantly decreased MMP and cellular ATP levels. However, the inhibition of the MMP by G-Rh2 was not consistent with that of ATP, with the inhibitory effect on MMP being stronger. This was probably due to the ATP produced in compensation by the glycolytic pathway. More active glycolysis takes place to compensate for the decreased ATP production due to impaired mitochondrial OXPHOS (41). During homeostasis, $70 \%$ of ATP is synthesized in the mitochondria; however, this percentage declines to $30 \%$ under a hypoxic environment (42). In the present study, it was also demonstrated that G-Rh2 does not completely inhibit glycolysis, exerting a stronger inhibitory 
effect of G-Rh2 on OXPHOS than on glycolysis in HeLa cells.

The overproduction of mtROS is a result of the events that inhibit the activity of ETC complexes (43). In fact, in the process of electron transfer from NADH to coenzyme Q in complex I, mtROS may be generated at the flavin mononucleotide site and the coenzyme $\mathrm{Q}$ binding site; the hemiquinone radical (QH-) of complex III produces mtROS by leaking electrons to $\mathrm{O} 2$ through a non-enzymatic reaction (17). Although ETC complex V (ATP synthase) is not a direct source of $\mathrm{mtROS}$, it participates in the regulation of energy metabolism, which in turn affects mtROS generation (44). In the present study, a suppressive effect of G-Rh2 on the activity of ETC complexes I, III and V was observed. To this end, the necessity of mtROS was further validated, which was stimulated by the inhibition of ETC complexes I, III and V in the induction of apoptosis. The results indicated that the overproduction of mtROS mediated by G-Rh2 was primarily responsible for the cytotoxicity caused by G-Rh2. After G-Rh2 treatment, cellular mtROS levels increased by $\sim 2.8$-fold and this was partially reversed by the mitochondrial target antioxidant Mito Q. Furthermore, Mito Q also inhibits apoptosis induced by G-Rh2. These results are in line with previous reports that suggested G-Rh2 induces apoptosis by promoting mtROS accumulation in human leukemia Jurkat, HepG2 and Hep3B cells $(34,36,45)$.

The molecular docking experiments indicated that due to powerful hydrogen bonding and/or hydrophobic interactions, G-Rh2 exhibited strong affinities towards ETC complex III $(-10.6 \mathrm{kcal} / \mathrm{mol})$, ETC complex I $(-7.73 \mathrm{kcal} / \mathrm{mol})$ and ETC complex V (-6.48 kcal/mol). Enzymatic activity experiments suggested that G-Rh2 significantly inhibited the activity of ETC complexes I, III and V and the degree of inhibition was basically the same. Conversely, the molecular docking results indicated that G-Rh2 has a higher affinity for ETC complex III. This implied that G-Rh2 may directly bind to ETC complex III and indirectly interfere with ETC complexes I and V. The binding sites of the molecular docking results suggested that post-translational modification of the protein is likely responsible for the ability of G-Rh2 to reduce the activity of the ETC complex enzymes under the condition that the protein expression of the representative subunits of complex I-V did not change. G-Rh2 may catalyze the phosphorylation of corresponding amino acids through hydrogen bonding.

To gain additional insight into the mechanism of action of ETC complex I, III and V in G-Rh2-induced mtROS-mediated apoptosis, overexpression vectors for NDUFS1, UQCRC1 and ATP5F1B, which are representative subunits of ETC complex I, III and V, respectively, were constructed. The results suggested that overexpression of UQCRC1 significantly attenuated G-Rh2-induced mtROS-mediated apoptosis in HeLa cells. Consistent with the molecular docking results, G-Rh2 mainly targets ETC complex III, which promotes the production of mtROS and ultimately induces apoptosis. It has been reported that ETC complex I and ETC complex III are closely connected through the interaction between protein subunits (46), which may be the cause of the decrease in ETC complex I activity. In addition, ETC complexes I-IV not only transfer electrons, but also establish the proton gradient of ETC complex V to synthesize ATP (17). The inhibition of ETC complex III hinders electron transfer and accumulated electrons may be responsible for the decrease of ETC complex V enzyme activity.

The limitation of the present study on G-Rh2 is that the experiments were all performed in cell lines cultured in vitro. It has been reported that G-Rh2 exhibits a variety of anti-tumor activities in vivo with no measurable toxicity even at a dose of $120 \mathrm{mg} / \mathrm{kg}$ (47), which means that G-Rh2 possesses a wide tolerance. However, due to the huge difference between in vitro and in vivo experiments, the actual mechanisms of G-Rh2 require to be further studied in vivo.

In conclusion, the present study indicated that G-Rh2 is a major contributor to apoptosis in HeLa cells through the mitochondrial pathway. In this process, G-Rh2 inhibits the activity of ETC complex III and indirectly affects the activity of ETC complexes I and V, leading to the overproduction of mtROS, decreased ATP synthesis and bidirectional inhibition of OXPHOS and glycolysis. To the best of our knowledge, the present study was the first to elucidate that the mechanism through which mtROS overproduction induces apoptosis in HPV-positive cervical cancer cells following G-Rh2 treatment was through inhibiting the activity of ETC complex III. This artificial regulation of mtROS at ETC complex III may be of great significance for cancer treatment.

\section{Acknowledgements}

Not applicable.

\section{Funding}

The research was supported by key projects at the central government level: The Ability Establishment of Sustainable use for Valuable Chinese Medicine Resources (grant no. 2060302), the National Key Research and Development Program of China (grant no. 2017YFC1702104) and the National Natural Foundation of China (grant no. 81703663).

\section{Availability of data and materials}

The datasets used and/or analyzed during the current study are available from the corresponding author upon reasonable request.

\section{Authors' contributions}

ML and JW conceived and designed the study. YL, SY, XX and JQ performed experiments. YY helped with the collection and assembly of data. YY, WZ and JQ analyzed the data and prepared the figures. ML, YL and WZ drafted and revised the manuscript. All authors read and approved the final manuscript. ML and WZ confirm the authenticity of all the raw data.

\section{Ethics approval and consent to participate}

Not applicable.

\section{Patient consent for publication}

Not applicable. 


\section{Competing interests}

The authors declare that they have no competing interests.

\section{References}

1. Wang R, Pan W, Jin L, Huang W, Li Y, Wu D, Gao C, Ma D and Liao S: Human papillomavirus vaccine against cervical cancer: Opportunity and challenge. Cancer Lett 471: 88-102, 2020.

2. Yang K, Park W, Huh SJ, Bae DS, Kim BG and Lee JW: Clinical outcomes in patients treated with radiotherapy after surgery for cervical cancer. Radiat Oncol J 35: 39-47, 2017.

3. Nokihara H, Lu S, Mok TSK, Nakagawa K, Yamamoto N, Shi YK, Zhang L, Soo RA, Yang JC, Sugawara S, et al: Randomized controlled trial of S-1 versus docetaxel in patients with non-small-cell lung cancer previously treated with platinum-based chemotherapy (East Asia S-1 Trial in Lung Cancer). Ann Oncol 28: 2698-2706, 2017.

4. Zhuang J, Yin J, Xu C, Mu Y and Lv S: 20(S)-Ginsenoside Rh2 induce the apoptosis and autophagy in U937 and K562 cells. Nutrients 10: 328, 2018.

5. Li X, Chu S, Lin M, Gao Y, Liu Y, Yang S, Zhou X, Zhang Y, $\mathrm{Hu}$ Y, Wang $\mathrm{H}$ and Chen $\mathrm{N}$ : Anticancer property of ginsenoside Rh2 from ginseng. Eur J Med Chem 203: 112627, 2020.

6. He XL, Xu XH, Shi JJ, Huang M, Wang Y, Chen X and Lu JJ: Anticancer effects of ginsenoside Rh2: A systematic review. Curr Mol Pharmacol: Mar 8, 2021 (Epub ahead of print).

7. Liu Y, Han M, Li X, Wang H, Ma M, Zhang S, Guo Y, Wang S, Wang Y, Duan N, et al: Age-related changes in the mitochondria of human mural granulosa cells. Hum Reprod 32: 2465-2473, 2017.

8. Wang YS, Lin Y, Li H, Li Y, Song Z and Jin YH: The identification of molecular target of (20S) ginsenoside Rh2 for its anti-cancer activity. Sci Rep 7: 12408, 2017.

9. Li B, Zhao J, Wang CZ, Searle J, He TC, Yuan CS and Du W: Ginsenoside Rh2 induces apoptosis and paraptosis-like cell death in colorectal cancer cells through activation of $\mathrm{p} 53$. Cancer Lett 301: 185-192, 2011

10. Zhu C, Liu F, Qian W, Zhang T and Li F: Combined effect of sodium selenite and ginsenoside Rh2 on HCT116 human colorectal carcinoma cells. Arch Iran Med 19: 23-29, 2016.

11. Xia T, Wang JC, Xu W, Xu LH, Lao CH, Ye QX and Fang JP: 20S-Ginsenoside Rh2 induces apoptosis in human Leukaemia Reh cells through mitochondrial signaling pathways. Biol Pharm Bull 37: 248-254, 2014

12. Cheng MH, Pan CY, Chen NF, Yang SN, Hsieh S, Wen ZH, Chen WF, Wang JW, Lu WH and Kuo HM: Piscidin-1 induces apoptosis via mitochondrial reactive oxygen species-regulated mitochondrial dysfunction in human osteosarcoma cells. Sci Rep 10: 5045, 2020.

13. Payen VL, Zampieri LX, Porporato PE and Sonveaux P: Proand antitumor effects of mitochondrial reactive oxygen species Cancer Metastasis Rev 38: 189-203, 2019.

14. Du X, Zhang P, Fu H, Ahsan HM, Gao J and Chen Q: Smart mitochondrial-targeted cancer therapy: Subcellular distribution, selective TrxR2 inhibition accompany with declined antioxidant capacity. Int J Pharm 555: 346-355, 2019.

15. Forman HJ and Kennedy JA: Role of superoxide radical in mitochondrial dehydrogenase reactions. Biochem Biophys Res Commun 60: 1044-1050, 1974

16. Baldissera MD, Souza CF, Grings M, Parmeggiani BS Leipnitz G, Moreira KLS, da Rocha MIUM, da Veiga ML, Santos RCV, Stefani LM and Baldisserotto B: Inhibition of the mitochondrial respiratory chain in gills of Rhamdia quelen experimentally infected by Pseudomonas aeruginosa: Interplay with reactive oxygen species. Microb Pathog 107: 349-353, 2017.

17. Zhao RZ, Jiang S, Zhang L and Yu ZB: Mitochondrial electron transport chain, ROS generation and uncoupling (Review). Int J Mol Med 44: 3-15, 2019.

18. Milkovic L, Cipak Gasparovic A, Cindric M, Mouthuy PA and Zarkovic N: Short Overview of ROS as cell function regulators and their implications in therapy concepts. Cells 8: 793, 2019.

19. Chen Q, Vazquez EJ, Moghaddas S, Hoppel CL and Lesnefsky EJ: Production of reactive oxygen species by mitochondria: Central role of complex III. J Biol Chem 278: 36027-36031, 2003.

20. Brand MD: The sites and topology of mitochondrial superoxide production. Exp Gerontol 45: 466-472, 2010
21. Fan W, Shen T, Ding Q, Lv Y, Li L, Huang K, Yan L and Song S: Zearalenone induces ROS-mediated mitochondrial damage in porcine IPEC-J2 cells. J Biochem Mol Toxicol: 31, 2017 doi: $10.1002 / \mathrm{jbt} .21944$

22. Wang HW, Zhang Y, Tan PP, Jia LS, Chen Y and Zhou BH Mitochondrial respiratory chain dysfunction mediated by ROS is a primary point of fluoride-induced damage in Hepa1-6 cells. Environ Pollut 255: 113359, 2019.

23. Li N, Ragheb K, Lawler G, Sturgis J, Rajwa B, Melendez JA and Robinson JP: Mitochondrial complex I inhibitor rotenone induces apoptosis through enhancing mitochondrial reactive oxygen species production. J Biol Chem 278: 8516-8525, 2003.

24. Liu Y, Wang J, Qiao J, Liu S, Wang S, Zhao D, Bai X and Liu M: Ginsenoside Rh2 inhibits HeLa cell energy metabolism and induces apoptosis by upregulating voltage-dependent anion channel 1. Int J Mol Med 46: 1695-1706, 2020.

25. Zhang $\mathrm{H}$, Gong J and Kong D: Induction of apoptosis and reversal of permeability glycoprotein-mediated multidrug resistance of MCF-7/ADM by ginsenoside Rh2. Int J Clin Exp Pathol 8: 4444-4456, 2015.

26. Bannon JH, Fichtner I, O'Neill A, Pampillón C, Sweeney NJ, Strohfeldt K, Watson RW, Tacke M and Mc Gee MM: Substituted titanocenes induce caspase-dependent apoptosis in human epidermoid carcinoma cells in vitro and exhibit antitumour activity in vivo. Br J Cancer 97: 1234-1241, 2007.

27. Mazumder MK, Paul R and Borah A: $\beta$-phenethylamine-a phenylalanine derivative in brain-contributes to oxidative stress by inhibiting mitochondrial complexes and DT-diaphorase: An in silico study. CNS Neurosci Ther 19: 596-602, 2013.

28. Kapur A, Beres T, Rathi K, Nayak AP, Czarnecki A, Felder M, Gillette A, Ericksen SS, Sampene E, Skala MC, et al: Oxidative stress via inhibition of the mitochondrial electron transport and Nrf-2-mediated anti-oxidative response regulate the cytotoxic activity of plumbagin. Sci Rep 8: 1073, 2018.

29. Chen LS, Nowak BJ, Ayres ML, Krett NL, Rosen ST, Zhang S and Gandhi V: Inhibition of ATP synthase by chlorinated adenosine analogue. Biochem Pharmacol 78: 583-591, 2009.

30. Emmanuel IA, Olotu FA, Agoni C and Soliman MES: Deciphering the 'Elixir of Life': Dynamic perspectives into the allosteric modulation of mitochondrial ATP synthase by J147, a novel drug in the treatment of Alzheimer's disease. Chem Biodivers 16: e1900085, 2019.

31. Larsen S, Nielsen J, Hansen CN, Nielsen LB, Wibrand F, Stride N, Schroder HD, Boushel R, Helge JW, Dela F and Hey-Mogensen M: Biomarkers of mitochondrial content in skeletal muscle of healthy young human subjects. J Physiol 590: 3349-3360, 2012.

32. Kim MJ, Yun H, Kim DH, Kang I, Choe W, Kim SS and Ha J: AMP-activated protein kinase determines apoptotic sensitivity of cancer cells to ginsenoside-Rh2. J Ginseng Res 38: 16-21, 2014.

33. Guo XX, Li Y, Sun C, Jiang D, Lin YJ, Jin FX, Lee SK and Jin YH: p53-dependent Fas expression is critical for Ginsenoside Rh2 triggered caspase- 8 activation in HeLa cells. Protein Cell 5: 224-234, 2014

34. Xia T, Wang J, Wang Y, Cai J, Wang M, Chen Q, Song J, Yu Z, Huang $\mathrm{W}$ and Fang J: Inhibition of autophagy potentiates anticancer property of 20 (S)-ginsenoside Rh2 by promoting mitochondria-dependent apoptosis in human acute lymphoblastic leukaemia cells. Oncotarget 7: 27336-27349, 2016.

35. Zare-Zardini H, Taheri-Kafrani A, Amiri A and Bordbar AK: New generation of drug delivery systems based on ginsenoside Rh2-, Lysine- and Arginine-treated highly porous graphene for improving anticancer activity. Sci Rep 8: 586, 2018.

36. Chen F, Deng Z, Xiong Z, Zhang B, Yang J and Hu J: A ROS-mediated lysosomal-mitochondrial pathway is induced by ginsenoside Rh2 in hepatoma HepG2 cells. Food Funct 6: 3828-3837, 2015.

37. Charlot JF, Prétet JL, Haughey C and Mougin C: Mitochondrial translocation of p53 and mitochondrial membrane potential (Delta Psi $\mathrm{m}$ ) dissipation are early events in staurosporine-induced apoptosis of wild type and mutated p53 epithelial cells. Apoptosis 9: 333-343, 2004.

38. Li Y, Qi H, Li X, Hou X, Lu X and Xiao X: A novel dithiocarbamate derivative induces cell apoptosis through p53-dependent intrinsic pathway and suppresses the expression of the E6 oncogene of human papillomavirus 18 in HeLa cells. Apoptosis 20: 787-795, 2015.

39. Seyfried TN, Arismendi-Morillo G, Mukherjee $P$ and Chinopoulos C: On the origin of ATP synthesis in cancer. iScience 23: 101761, 2020. 
40. Solsona-Vilarrasa E, Fucho R, Torres S, Nuñez S, Nuño-Lámbarri $\mathrm{N}$, Enrich $\mathrm{C}$, García-Ruiz $\mathrm{C}$ and Fernández-Checa JC: Cholesterol enrichment in liver mitochondria impairs oxidative phosphorylation and disrupts the assembly of respiratory supercomplexes. Redox biology 24: 101214, 2019.

41. Cappelli E, Cuccarolo P, Stroppiana G, Miano M, Bottega R Cossu V, Degan P and Ravera S: Defects in mitochondrial energetic function compels Fanconi Anaemia cells to glycolytic metabolism. Biochim Biophys Acta Mol Basis Dis 1863: 1214-1221, 2017.

42. Jaña F, Faini F, Lapier M, Pavani M, Kemmerling U, Morello A, Maya JD, Jara J, Parra E and Ferreira J: Tumor cell death induced by the inhibition of mitochondrial electron transport: The effect of 3-hydroxybakuchiol. Toxicol Appl Pharmacol 272: 356-364, 2013.

43. Lindsay DP, Camara AK, Stowe DF, Lubbe R and Aldakkak M: Differential effects of buffer $\mathrm{pH}$ on $\mathrm{Ca}(2+)$-induced ROS emission with inhibited mitochondrial complexes I and III. Front Physiol 6: 58, 2015.

44. Kaludercic N and Giorgio V: The dual function of reactive Oxygen/Nitrogen species in bioenergetics and cell death: The role of ATP synthase. Oxid Med Cell Longev 2016: 3869610 , 2016.
45. Park HM, Kim SJ, Kim JS and Kang HS: Reactive oxygen species mediated ginsenoside $\mathrm{Rg} 3$ - and $\mathrm{Rh} 2$-induced apoptosis in hepatoma cells through mitochondrial signaling pathways. Food Chem Toxico 50: 2736-2741, 2012.

46. Gu J, Wu M, Guo R, Yan K, Lei J, Gao N and Yang M: The architecture of the mammalian respirasome. Nature 537: 639-643, 2016.

47. Musende AG, Eberding A, Wood C, Adomat H, Fazli L, Hurtado-Coll A, Jia W, Bally MB and Guns ET: Pre-clinical evaluation of Rh2 in PC-3 human xenograft model for prostate cancer in vivo: Formulation, pharmacokinetics, biodistribution and efficacy. Cancer Chemother Pharmacol 64: 1085-1095, 2009. International (CC BY-NC-ND 4.0) License. 